University of Nebraska - Lincoln

DigitalCommons@University of Nebraska - Lincoln

8-1-2006

\title{
Hybrid atomistic-coarse-grained treatment of multiscale processes in heterogeneous materials: A self-consistent-field approach
}

\author{
Dennis J. Diestler \\ University of Nebraska-Lincoln, ddiestler1@unl.edu \\ H. Zhou \\ University of Nebraska-Lincoln \\ Ruqiang Feng \\ University of Nebraska-Lincoln, rfeng1@unl.edu \\ Xiao Cheng Zeng \\ University of Nebraska-Lincoln, xzeng1@unl.edu
}

Follow this and additional works at: https://digitalcommons.unl.edu/chemzeng

Part of the Chemistry Commons

Diestler, Dennis J.; Zhou, H.; Feng, Ruqiang; and Zeng, Xiao Cheng, "Hybrid atomistic-coarse-grained treatment of multiscale processes in heterogeneous materials: A self-consistent-field approach" (2006). Xiao Cheng Zeng Publications. 4.

https://digitalcommons.unl.edu/chemzeng/4

This Article is brought to you for free and open access by the Published Research - Department of Chemistry at DigitalCommons@University of Nebraska - Lincoln. It has been accepted for inclusion in Xiao Cheng Zeng Publications by an authorized administrator of DigitalCommons@University of Nebraska - Lincoln. 


\title{
Hybrid atomistic-coarse-grained treatment of multiscale processes in heterogeneous materials: A self-consistent-field approach
}

\author{
D. J. Diestler ${ }^{\mathrm{a})}$ \\ Department of Agronomy and Horticulture, University of Nebraska-Lincoln, Lincoln, Nebraska 68583 \\ H. Zhou and R. Feng \\ Department of Engineering Mechanics, University of Nebraska-Lincoln, Lincoln, Nebraska 68588 \\ X. C. Zeng \\ Department of Chemistry, University of Nebraska-Lincoln, Lincoln, Nebraska 68588
}

(Received 15 May 2006; accepted 28 June 2006; published online 10 August 2006)

\begin{abstract}
A treatment of multiscale quasistatic processes that combines an atomistic description of microscopic heterogeneous ("near") regions of a material with a coarse-grained (quasicontinuum) description of macroscopic homogeneous ("far") regions is presented. The hybrid description yields a reduced system consisting of the original atoms of the near regions plus pseudoatoms (nodes of the coarse-graining mesh) of the far regions, which interact through an effective many-body potential energy $V_{\text {eff }}$ that depends on the thermodynamic state. The approximate nature of $V_{\text {eff }}$ gives rise to "ghost forces," which are reflected in spurious heterogeneities close to interfaces between near and far regions. The impact of ghost forces, which afflict all previous hybrid schemes, is greatly diminished by a self-consistent-field hybrid atomistic-coarse-grained (SCF-HACG) methodology. Tests of the SCF-HACG technique on a fully three-dimensional prototypal model [Lennard-Jones $(12,6)$ crystal] yield thermomechanical properties (e.g., local stress) in good agreement with "exact" properties computed in the fully atomistic limit. The SCF-HACG method is also successfully used to characterize the grain boundary in a Lennard-Jones bicrystal. () 2006 American Institute of Physics. [DOI: 10.1063/1.2234776]
\end{abstract}

\section{INTRODUCTION}

Such practically important phenomena as the evolution of defects in materials and thin-film lubrication are inherently multiscale because they entail the dynamic coupling between microscopic heterogeneous ("near") regions and macroscopic homogeneous ("far") regions. A reliable description of the behavior of the whole system dictates an atomistic treatment of the near regions, whereas the far regions can be handled adequately on the continuum scale. Several schemes that meld an atomistic description of the near regions with a continuum description of the far regions have been proposed since the early 1990s. ${ }^{1-8}$ These have been reviewed ad nauseum in recent years. ${ }^{9-12}$

\section{A. Quasicontinuum method}

Introduced by Tadmor et al., ${ }^{2}$ the quasicontinuum (QC) method was devised to treat quasistatic propagation of defects in crystals at absolute zero $(T=0 \mathrm{~K})$. The QC method coarse-grains the whole system by covering the crystal lattice with a finite-element mesh. Coarse graining introduces constraints among the atomic coordinates, thereby greatly reducing the number of degrees of freedom. The myriad original atoms are replaced by relatively few pseudoatoms that interact via a coarse-grained many-body potential energy $U_{c}\left(\mathbf{r}^{N_{n}}\right)$, which is regarded as a functional of the $3 N_{n}$-dimensional

\footnotetext{
${ }^{a)}$ Electronic mail: ddiestler1@unl.edu
}

nodal configuration $\mathbf{r}^{N_{n}}$, where $N_{n}$ is the number of nodes. Under given boundary conditions, which correspond to a prescribed application of strains or stresses, $U_{c}$ is minimized. This procedure yields the optimum nodal configuration.

\section{B. Local versus nonlocal elements}

The coarse-grained potential energy is expressed as a sum of contributions from individual finite elements, which can be categorized according to their dimensions relative to the range $r_{c}$ of interactions between atoms. In the relatively vast far regions, where the crystal is deformed only slightly from the reference configuration, elements with dimensions much greater than $r_{c}$ can be employed. The contribution of such a large element to $U_{c}$ is computed as if the atomic lattice underlying it were part of an infinite lattice deformed from the reference configuration in the same manner. (Throughout this article we take all elements to be constantstrain elements, that is the lattice underlying any element is homogeneously distorted as its nodes are displaced from a given reference configuration. All of the underlying atoms occupying the lattice sites move in unison and their positions can be calculated from a knowledge of solely the nodal positions.) Such a large element is called "local" because its contribution to $U_{c}$ depends on only the positions of its own nodes. As one moves from far (uniform) regions toward near (nonuniform) regions, the elements must gradually become smaller so that the increasing heterogeneity can be described. When the dimensions of an element approach $r_{c}$ it becomes 
"nonlocal" in that its contribution to $U_{c}$ depends on both its own nodal configuration and those of neighboring elements whose underlying atoms lie within a distance $r_{c}$ from a "reference" atom under the nonlocal element. In the limit where the dimensions of all elements equal the lattice constant (every atom is a node) the expression for $U_{c}$ is identical with that based directly on the atomistic interactions. Thus, the multiple scales are seamlessly merged.

\section{Thermal effects}

Depending on the thermodynamic state, the thermal contribution to the stress can become significant already when the thermal energy of an atom $\left(\sim k_{B} T\right.$, where $k_{B}$ is Boltzmann's constant) is only a relatively small fraction of its binding energy in the crystal. ${ }^{13}$ Hence, the $T=0 \mathrm{~K}$ restriction on the original QC method becomes critical. Over the past decade or so we have been exploring ways of incorporating thermal effects in the QC technique. Our first naïve idea was simply to require that the nodal configurations satisfy the Boltzmann distribution. ${ }^{14}$ We accomplish this by implementing $U_{c}$ in a standard Monte Carlo (MC) computer simulation. ${ }^{15}$ However, when applied to a two-dimensional (2D) Lennard-Jones $(12,6)$ crystal, this crude treatment leads to errors in the diagonal components of the ("global") stress tensor proportional to $\left(N-N_{n}\right)$, which is just the number of nonnodal atoms underlying the finite-element mesh, when the mesh is so constructed that nodes coincide with atoms. The error is due to neglect of thermal motion of the underlying (non-nodal) atoms. Their random movements are quenched by the dynamical constraint that they move in concert with the nodes.

We have tried two alternative approaches that eschew the dynamical constraint on underlying atoms. The first of these is a precise analog of the original QC method: $U_{c}$ is just replaced with a free-energy functional $F_{c}\left(\mathbf{r}^{N_{n}}\right)$ appropriate to the thermodynamic state variables that are controlled in the quasistatic process of interest. ${ }^{13}$ This coarse-grained free-energy functional technique applied to a fully threedimensional (3D) Lennard-Jones $(12,6)$ crystal at fixed density and temperature yields stress components in excellent agreement with "exact" results (obtained from conventional atomic-scale computer simulation).

In a second alternative approach ${ }^{16}$ conceived to avoid dynamically constraining the underlying atoms, we require that the nodes of the coarse-graining mesh coincide with atoms. The original atoms are consequently separated into two groups: the nodal atoms and the non-nodal underlying atoms. We then formally carry out the integrations over the phase space of the underlying subsystem in the canonical partition function for the whole system. This procedure yields an effective potential energy $V_{\text {eff }}\left(\mathbf{r}^{N_{n}}\right)$ for the nodal subsystem that depends on the thermodynamic state. $V_{\text {eff }}$ comprises two contributions: the potential energy $U_{c}\left(\mathbf{r}^{N_{n}}\right)$ of the dynamically constrained system and the Helmholtz energy of the underlying subsystem in the field of the nodal subsystem in a given (instantaneous) configuration $\mathbf{r}^{N_{n}}$. The free-energy component, which is given as a sum of contributions from individual elements by analogy with $U_{c}$, accounts precisely for the thermal motion of the underlying atoms that is suppressed in the dynamically constrained treatment. We employed $V_{\text {eff }}$ in a MC simulation of the 2D Lennard-Jones $(12,6)$ crystal, finding excellent agreement between coarsegrained and exact results for the stress for a selection of thermodynamic states. ${ }^{16}$

\section{Hybrid atomistic-coarse-grained treatment}

All of the above-described approaches suffer from another limitation of the QC method, which is that it is inconvenient to treat processes involving diffusion in the near regions. To handle such situations we previously proposed a hybrid atomistic-coarse-grained (HACG) technique. The key idea behind the HACG scheme is to coarse-grain only the far regions, which behave essentially as elastic solid for the duration of the process of interest. ${ }^{17}$ We applied the dynamically constrained version of the HACG to reversible shearing of a 2D model tribological contact: two planar hard Lennard-Jones $(12,6)$ substrates that sandwich a softer Lennard-Jones film. ${ }^{17,18}$ Constant-load MC simulations yield shear-stress profiles (plots of "global" shear stress versus shear strain) in good agreement with the exact profiles (obtained from parallel atomic-scale simulations), but mean separation profiles (i.e., plots of the mean gap between the substrates versus shear strain) that disagree with the exact separation profiles. The discrepancy is again due to the neglect of the underlying non-nodal thermal motion, the absence of which suppresses thermal expansion of the substrates. Additional MC simulations ${ }^{19}$ of this $2 \mathrm{D}$ model that include the neglected free-energy contribution give good agreement between HACG and exact separation profiles.

\section{E. Self-consistent-field HACG treatment: Local properties}

The HACG methodology has thus far been employed to compute only the global properties of the system (i.e., properties of the system considered as a whole), which are properties of the kind commonly measured. In order to characterize the system more completely, one can usefully define local properties, that is, properties associated with specific subsystems (e.g., atoms or finite elements) of the whole system. As it is currently implemented, however, the HACG treatment $^{17,18}$ is incapable of yielding reliable local properties. One of the main points of this article is to introduce a modified implementation, that is, the self-consistent-field (SCF-) HACG treatment, which not only accounts for thermal effects in the coarse-grained far regions but at once permits the dependable computation of local properties.

To test the SCF-HACG scheme properly, we must first apply it to a system whose thermomechanical properties are either known or can be determined by independent methods. We adopt as a prototypal test system a Lennard-Jones $(12,6)$ crystal that is partitioned into three slabs. The outer two slabs play the role of (coarse-grained) far regions and the inner slab that of the (atomistic) near region. A correct description of this system in a state of thermodynamic equilibrium should give uniformly constant local properties, since every identical subsystem (e.g., atom) is subject to the same envi- 


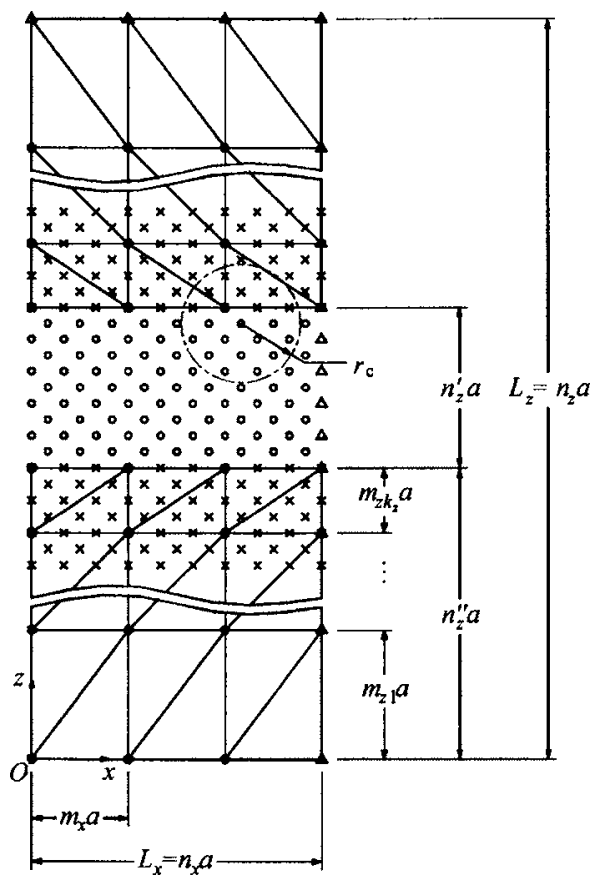

FIG. 1. Schematic side view (in positive $y$ direction) of Lennard-Jones $(12,6)$ crystal. Atoms depicted in equilibrium positions at $T=0 \mathrm{~K}$ in near region (inner slab) by open circles and in far regions (outer slabs) by crosses; nodes in far regions represented by filled circles. The open triangles on top and right boundaries signify periodic images of atoms (nodes).

ronment. However, the partitioning gives rise to false heterogeneities in the vicinity of the two near-far interfaces. These are manifested as discontinuities in the local stress, as computed in the usual implementation ${ }^{17}$ of the HACG scheme (see Sec. V). The errors in local stress can be traced to "ghost forces," "10,20 which are engendered by asymmetric contributions of atoms and finite elements to the coarse-grained effective potential energy.

The SCF-HACG treatment represents the culmination of a lengthy search for an antidote to ghost forces. In the new approach, a MC cycle comprises two stages: (1) random trial displacements of the near-region atoms in the "external" field of the frozen far regions and (2) random trial moves of the far-region nodes in the external field of the frozen near region. The potential energy governing the acceptance of trial moves is chosen so as to reduce the offending asymmetric contributions. As the number of MC cycles increases, alternating between the two stages, each region relaxes in the external field due to its frozen counterpart. The whole system thus relaxes self-consistently. In this fashion the impact of ghost forces is mollified.

\section{PROTOTYPAL TEST MODEL}

\section{A. Microscopic description}

We simplify the analysis from the outset by restricting consideration to the ideal model that we employ to test the new SCF-HACG technique (see Sec. VI). Schematized in Fig. 1, the system consists of an $L_{x} \times L_{y} \times L_{z}$ rectangular prism of Lennard-Jonesium $(12,6)$. The potential (configurational) energy $U$ can be expressed as

$$
U\left(\mathbf{r}_{1}, \mathbf{r}_{2}, \ldots, \mathbf{r}_{N}\right)=\sum_{i=1}^{N} u_{i},
$$

where $\mathbf{r}_{i}$ stands for the position of atom $i$ and $N$ is the total number of atoms. The contribution $u_{i}$ of atom $i$ is given by

$$
u_{i}=\frac{1}{2} \sum_{j \neq i} \phi\left(r_{i j}\right), \quad r_{i j}<r_{c}
$$

where $\phi \equiv 4 \varepsilon\left[(\sigma / r)^{12}-(\sigma / r)^{6}\right], \mathbf{r}_{i j} \equiv \mathbf{r}_{i}-\mathbf{r}_{j}$ is the position of atom $i$ with respect to atom $j$, and $r_{i j}=\left|\mathbf{r}_{i j}\right|$ is the distance between atoms $i$ and $j$. In practice, the summation on $j$ in Eq. (2.2) is restricted by the inequality $r_{i j}<r_{c}$, where $r_{c}$ is the radius of a "cutoff" sphere centered on atom $i$. The Hamiltonian is

$$
H=\sum_{i=1}^{N} p_{i}^{2} / 2 m+U\left(\mathbf{r}_{1}, \mathbf{r}_{2}, \ldots, \mathbf{r}_{N}\right) .
$$

Here $\mathbf{p}_{i}=m \dot{\mathbf{r}}_{i}$ is the momentum conjugate to the position $\mathbf{r}_{i}$ of atom $i, m$ is the mass of an atom, and the dot over the symbol stands for the (total) derivative with respect to time.

A side view of the face-centered-cubic (fcc) structure of the crystal in Fig. 1 depicts the atoms in their equilibrium positions at $T=0 \mathrm{~K}$ for the given density. This is the reference configuration used in the computer simulations described in Secs. V and VI. Note that in the reference configuration every atom is subject to the same environment as every other atom. All $u_{i}$ [see Eq. (2.2)] are therefore equal and $U=N u_{0}$, where $u_{0}$ is the potential energy per atom.

To eliminate extraneous effects due to surfaces and edges of the prism, we impose periodic boundary conditions on the faces of the prisms in the $x, y$, and $z$ directions. There are $n_{\alpha}$ unit cells along the $\alpha$ direction $(\alpha=x, y, z) ; L_{\alpha}=n_{\alpha} a$, where $a$ is the cell constant. Thus, the total number of atoms is $N=4 n_{x} n_{y} n_{z}$ and the number density is $\rho=N / V=4 / a^{3}$.

\section{B. Thermodynamics}

The controlled thermodynamic state variables are the side lengths $L_{\alpha}$, the absolute temperature $T$, and the number of atoms $N$. For given fixed values of these independent state variables the Helmholtz energy $F=E-T S$ (where $E$ is the internal energy and $S$ is the entropy) is minimum when the system is in a state of thermodynamic equilibrium. Gibbs's fundamental relation governing quasistatic (reversible) transformations of the system is

$$
d F=-S d T+\mu d N+d W,
$$

where $\mu$ stands for the chemical potential and $d W$ for the mechanical work done by the system on the surroundings. If we restrict our attention to work resulting from homogeneous expansion or contraction of the crystal, then $d W$ can be written as

$$
d W=\tau_{x x} L_{y} L_{z} d L_{x}+\tau_{y y} L_{x} L_{z} d L_{y}+\tau_{z z} L_{x} L_{y} d L_{z},
$$

where $\tau_{\alpha \alpha}$, the $\alpha \alpha$ component of the (global) stress tensor, is the mean force per unit area applied to the surface of the crystal that points in the $\alpha$ direction. From Eqs. (2.4) and (2.5) we deduce 


$$
\tau_{x x}=\frac{1}{L_{y} L_{z}}\left(\frac{\partial F}{\partial L_{x}}\right)_{T, N, L_{y}, L_{z}}
$$

and similar relations for $\tau_{y y}$ and $\tau_{z z}$. We define the global mean stress by

$$
\bar{\tau}=\frac{1}{3} \sum_{\alpha} \tau_{\alpha \alpha}
$$

\section{Coarse graining}

The crystal is partitioned into three slabs by the planes $z=n_{z}^{\prime \prime} a$ and $z=\left(n_{z}^{\prime \prime}+n_{z}^{\prime}\right) a$ so that the outer two slabs are of the same thickness $n_{z}^{\prime \prime} a$ ( $n_{z}^{\prime \prime}$ is the number of layers of unit cells comprised by the outer slabs and $n_{z}^{\prime}$ is the number of layers of unit cells composing the inner slab, see Fig. 1). Each of the outer slabs is then further divided into rectangular prisms by planes normal to the coordinate axes. The division is symmetrical about the midplane $z=L_{z} / 2$. In the lateral $(x, y)$ directions the partitioning planes $\left[x=m_{x} a, x=2 m_{x} a, \ldots x=\left(n_{x}\right.\right.$ $\left.-m_{x}\right) a ; y=m_{y} a, y=2 m_{y} a, \ldots y=\left(n_{y}-m_{y}\right) a$, where $n_{x}$ and $n_{y}$ are integer multiples of $m_{x}$ and $m_{y}$, respectively, i.e., $n_{x}$ $=k_{x} m_{x}$ and $\left.n_{y}=k_{y} m_{y}\right]$ create $\left(k_{x} k_{y}\right) m_{x} a \times m_{y} a \times n_{z}^{\prime \prime} a$ rectangular prisms in each outer slab. Planes perpendicular to the $z$ axis $\quad\left[z=m_{z 1} a, \quad z=\left(m_{z 1}+m_{z 2}\right) a, \ldots z=\sum_{j=1}^{k_{z}-1} m_{z j} a ; \quad z=\left(n_{z}\right.\right.$ $\left.-\sum_{j=1}^{k_{z}-1} m_{z j}\right) a, \ldots z=\left(n_{z}-m_{z 1}\right) a$, where $k_{z}$ is the number of divisions of the outer slabs] divide these prisms into yet smaller "subprisms" of varying $z$ dimension. The set of integers $\left\{m_{z i}, i=1,2, \ldots k_{z}\right\}$ satisfies the constraint $\sum_{i=1}^{k_{z}} m_{z i}=n_{z}^{\prime \prime}$. Finally, we coarse-grain the lattice under each of these subprisms by inscribing six tetrahedral finite elements. The nodes of the resulting mesh coincide with atoms at the vertices of the subprisms (see Fig. 1). The total number of subprisms is $2 k_{x} k_{y} k_{z}$. Therefore the number of elements in the whole mesh is $N_{e}=12 k_{x} k_{y} k_{z}$. Because of the periodic boundary conditions the number of independent nodes is $k_{x} k_{y}\left(2 k_{z}\right.$ +1 ). Likewise, the number of atoms (not all dynamically independent) contained by the whole system is $4 n_{x} n_{y} n_{z}: N_{b}^{\prime \prime}$ $=2 n_{x} n_{y}\left(2 n_{z}^{\prime \prime}+1\right)$ in the bottom (outer) slab; $N^{\prime}=2 n_{x} n_{y}\left(2 n_{z}^{\prime}\right.$ -1) in the inner slab; $N_{t}^{\prime \prime}=4 n_{x} n_{y} n_{z}^{\prime \prime}$ in the top (outer) slab. The total number of atoms underlying the finite-element mesh is $N^{\prime \prime}=N_{b}^{\prime \prime}+N_{t}^{\prime \prime}$.

Because we assume all elements are constant-strain elements, the (equilibrium) position $\mathbf{r}_{i}$ of atom $i$ under element $e$ is given in terms of the positions $\left\{\mathbf{R}_{k}^{e}, k=1,2,3,4\right\}$ of the nodes of $e$ by

$$
\mathbf{r}_{i}=\sum_{k=1}^{4} \mathbf{R}_{k}^{e} N_{k}^{e}(i)
$$

where the $N_{k}^{e}(i)$ are linear interpolation functions that depend on the reference configuration. ${ }^{21}$ Hence, we can express the atomic configuration of the whole coarse-grained subsystem economically in terms of the nodal configuration as

$$
\mathbf{r}_{\alpha}^{\left(N^{\prime \prime}\right)}=\mathbf{d R}_{\sigma}^{\left(N_{n}\right)},
$$

where $\mathbf{r}_{\alpha}^{\left(N^{\prime \prime}\right)}$ is the $N^{\prime \prime} \times 1$ matrix of atomic $\alpha(=x, y, z)$ coordinates and $\mathbf{R}_{\alpha}^{\left(N_{n}\right)}$ is the $N_{n} \times 1$ matrix of nodal $\alpha$ coordinates.
The elements of the $N^{\prime \prime} \times N_{n}$ matrix $\mathbf{d}$ can be obtained from the relation in Eq. (2.8).

\section{EFFECTIVE POTENTIAL ENERGY OF THE COARSE-GRAINED SYSTEM}

The classical-limit canonical partition function $Q$, which provides the bridge between microscopic and macroscopic descriptions of the system, is given formally by

$$
Q=h^{-3 N} \int d \mathbf{p}^{N} \int d \mathbf{r}^{N} \exp \left(-H / k_{B} T\right) .
$$

In Eq. (3.1) $H$ stands for the Hamiltonian [see Eq. (2.3)], $\mathbf{r}^{N}=\left(\mathbf{r}_{1}, \mathbf{r}_{2}, \ldots, \mathbf{r}_{N}\right)$ for the collection of positions of the atoms, $\mathbf{p}^{N}=\left(\mathbf{p}_{1}, \mathbf{p}_{2}, \ldots, \mathbf{p}_{N}\right)$ for the collection of conjugate momenta, and $h$ for Planck's constant.

The partial coarse graining described above in Sec. II C divides the atoms of the prototypal test model into two subsets: primary atoms, which comprise the nodal atoms plus the atoms of the near region, which do not lie under the finite-element mesh; and secondary atoms, which are the non-nodal atoms underlying the mesh.

\section{A. Harmonic approximation}

We now expand the configurational energy $U\left(\mathbf{r}^{N}\right)$ in Taylor's series about the "minimum" configuration of secondary atoms, keeping the primary atoms fixed in a given configuration. In other words, we expand $U$ about the configuration $\mathbf{r}_{0}^{N_{q}}$ of secondary atoms in which $U$ is minimum when the primary atoms are fixed in an arbitrary, but admissible, configuration $\mathbf{r}^{N_{p}}$, where $N_{p}$ and $N_{q}$ are the respective numbers of primary and secondary atoms. Thus, we obtain

$$
\begin{aligned}
U\left(\mathbf{r}^{N}\right)= & U\left(\mathbf{r}^{N_{p}}, \mathbf{r}^{N_{q}}\right) \\
= & U\left(\mathbf{r}^{N_{p}}, \mathbf{0}\right)+\sum_{i=1}^{N_{q}} \sum_{\alpha}\left(\partial U / \partial r_{i \alpha}\right)_{0} \Delta r_{i \alpha} \\
& +\frac{1}{2} \sum_{i=1}^{N_{q}} \sum_{\alpha} \sum_{j=1}^{N_{q}} \sum_{\beta} \Delta r_{i \alpha}\left(\partial^{2} U / \partial r_{i \alpha} \partial r_{j \beta}\right)_{0} \Delta r_{j \beta}+\cdots,
\end{aligned}
$$

where we neglect terms of degree higher than the second (i.e., we make the harmonic approximation). In Eq. (3.2) the subscript $\mathbf{0}$ denotes the equilibrium (minimum) configuration $\mathbf{r}_{0}^{N_{q}}$ of secondary atoms, $\alpha$ and $\beta$ label Cartesian components, and $\Delta r_{i \alpha}=r_{i \alpha}-r_{i \alpha}^{0}$ is the $\alpha$ component of the displacement of atom $i$ from its equilibrium position. By definition, the forces on the secondary atoms vanish in the equilibrium configuration, where $U$ is minimum with the fixed primary-atom configuration. We can then recast Eq. (3.2) succinctly as

$$
U\left(\mathbf{r}^{N}\right)=U\left(\mathbf{r}^{N_{p}}, \mathbf{r}^{N_{q}}\right)=U\left(\mathbf{r}^{N_{p}}, \mathbf{0}\right)+\frac{1}{2} \Delta \mathbf{x}^{T} \cdot \boldsymbol{\kappa}\left(\mathbf{r}^{N_{p}}\right) \cdot \Delta \mathbf{x},
$$

where $\Delta \mathbf{x}$ stands for the $3 N_{q} \times 1$ matrix of Cartesian components of secondary-atom displacements, $\boldsymbol{\kappa}$ for the $3 N_{q}$ $\times 3 N_{q}$ matrix of "force constants," and $T$ for the transpose. Note that $\boldsymbol{\kappa}$ depends implicitly on the primary-atom configuration. Substituting Eqs. (2.3) and (3.3) into Eq. (3.1) and 
performing the integrations over the primary momenta, we get

$$
Q=\Lambda^{-3 N_{p}} \int d \mathbf{r}^{N_{p}} \exp \left\{-\left[U\left(\mathbf{r}^{N_{p}}, \mathbf{0}\right)+F_{q}\left(\mathbf{r}^{N_{p}}\right)\right] / k_{B} T\right\}
$$

where $\Lambda \equiv\left(h^{2} / 2 \pi m k_{B} T\right)^{1 / 2}$ and

$$
F_{q}\left(\mathbf{r}^{N_{p}}\right) \equiv-k_{B} T \ln Q_{q}\left(\mathbf{r}^{N_{p}}\right)
$$

is the Helmholtz energy of the secondary subsystem in the field of the primary subsystem in configuration $\mathbf{r}^{N_{p}}$. The corresponding canonical partition function of the secondary subsystem is

$$
\begin{aligned}
Q_{q}\left(\mathbf{r}^{N_{p}}\right)= & h^{-3 N_{q}} \int d \mathbf{p}^{N_{q}} \int d \mathbf{r}^{N_{q}} \exp \left\{-\left[\sum_{i=1}^{N_{q}} p_{i}^{2} / 2 m\right.\right. \\
& \left.\left.+\frac{1}{2} \Delta \mathbf{x}^{T} \cdot \boldsymbol{\kappa}\left(\mathbf{r}^{N_{p}}\right) \cdot \Delta \mathbf{x}\right] / k_{B} T\right\} .
\end{aligned}
$$

We can write $Q_{q}$ in terms of the normal-mode frequencies $\Omega_{i}$ as

$$
Q_{q}\left(\mathbf{r}^{N_{p}}\right)=\prod_{i=1}^{3 N_{q}} k_{B} T / \hbar \Omega_{i}\left(\mathbf{r}^{N_{p}}\right)
$$

where $\hbar \equiv h / 2 \pi$. The normal-mode frequencies are obtained in principle by a unitary transformation of the dynamical matrix $\mathbf{D}\left(\mathbf{r}^{N_{p}}\right) \equiv m^{-1} \boldsymbol{\kappa}\left(\mathbf{r}^{N_{p}}\right)$,

$$
\mathbf{U}^{T} \mathbf{D} \mathbf{U}=\left(\mathbf{\Omega}^{2}\right)_{d},
$$

where the subscript $d$ signifies that $\boldsymbol{\Omega}^{2}$ is diagonal. Combining Eqs. (3.5) and (3.7), we obtain the effective potential energy

$$
\begin{aligned}
& V_{\mathrm{eff}}\left(\mathbf{r}^{N_{p}}\right) \equiv U\left(\mathbf{r}^{N_{p}}, \mathbf{0}\right)+F_{q}\left(\mathbf{r}^{N_{p}}\right) \\
& =U\left(\mathbf{r}^{N_{p}}, \mathbf{0}\right)+k_{B} T \sum_{k=1}^{3 N_{q}} \ln \left[\hbar \Omega_{k}\left(\mathbf{r}^{N_{p}}\right) / k_{B} T\right],
\end{aligned}
$$

which depends on the thermodynamic state.

\section{B. Local harmonic approximation}

Though the expression for $V_{\text {eff }}$ in Eq. (3.9) is exact (within the harmonic approximation), it is impractical, since we cannot afford to compute all normal-mode frequencies of the secondary subsystem for all admissible primary-atom configurations that must be generated in the MC simulations (see Sec. V). We therefore resort to the local harmonic approximation, in which the atoms are taken to be independent of one another (i.e., each atom is assumed to vibrate about its equilibrium position as if its neighbors were fixed in their equilibrium positions). ${ }^{22}$ In this approximation $\mathbf{D}$ is block diagonal and Eq. (3.8) can be recast as

$$
\mathbf{U}_{i}^{T} \mathbf{D}_{i} \mathbf{U}_{i}=\left(\boldsymbol{\Omega}_{i}^{2}\right)_{d}, \quad i=1,2, \ldots, N_{q},
$$

where the subscript $i$ refers to the $i$ th atom and all matrices are $3 \times 3$. Then $F_{q}$ can be rewritten as

$$
F_{q}\left(\mathbf{r}^{N_{p}}\right)=k_{B} T \sum_{i=1}^{N_{q}} \ln \left[\prod_{\lambda=1}^{3} \hbar \Omega_{i \lambda} / k_{B} T\right]
$$

where $\left\{\Omega_{i \lambda}, \lambda=1,2,3\right\}$ are the three local normal-mode frequencies associated with atom $i$. We can write the product of local normal-mode frequencies appearing in Eq. (3.11) as

$$
\prod_{\lambda} \Omega_{i \lambda}=\left[\operatorname{det}\left(\boldsymbol{\Omega}_{i}^{2}\right)_{d}\right]^{1 / 2}=\left\{\operatorname{det}\left[\mathbf{U}_{i}^{T} \mathbf{D}_{i} \mathbf{U}_{i}\right]\right\}^{1 / 2}=\left[\operatorname{det} \mathbf{D}_{i}\right]^{1 / 2},
$$

where the last equality follows because $\mathbf{U}_{i}$ is unitary (i.e., $\mathbf{U}_{i}^{T}=\mathbf{U}_{i}^{-1}$ ). Substitution of Eq. (3.12) into Eq. (3.11) yields

$$
F_{q}\left(\mathbf{r}^{N_{p}}\right)=3 k_{B} T \sum_{i=1}^{N_{q}} \ln \left[\hbar\left(\operatorname{det} \mathbf{D}_{i}\right)^{1 / 6} / k_{B} T\right] .
$$

In the case of a perfect crystal this expression simplifies to

$$
F_{q}\left(\mathbf{r}^{N_{p}}\right)=3 N_{q} k_{B} T \ln \left[\hbar(\operatorname{det} \mathbf{D})^{1 / 6} / k_{B} T\right]
$$

since all atoms are equivalent. The local dynamical matrix $\mathbf{D}$ is given by

$$
\text { (D) })_{\alpha \beta}=m^{-1}\left(\frac{\partial^{2} U}{\partial r_{i \alpha} \partial r_{i \beta}}\right)_{0}=m^{-1}\left\{\frac{\partial^{2}}{\partial r_{i \alpha} \partial r_{i \beta}}\left[\sum_{j \neq l} \phi\left(r_{i j}\right)\right]\right\}_{0},
$$

where $i$ denotes an atom of the infinite crystal that serves as a reference and the summation on $j$ includes all neighbors of $i$ that lie within the cutoff sphere of radius $r_{c}$ centered on $i$. An analytical expression for $D_{\alpha \beta}$ is derived in Appendix B.

\section{Coarse-grained formula for the effective potential energy}

Since the deformation of a constant-strain element is homogeneous, which implies that the lattice underlying the element is that of a slightly strained perfect crystal, we can express the dynamically constrained contribution to $V_{\text {eff }}$ [see Eq. (3.9)] as

$$
U\left(\mathbf{r}^{N_{p}}, \mathbf{0}\right)=\sum_{i=1}^{N^{\prime}} u_{i}+\sum_{e=1}^{N_{e}} N_{\alpha}^{e} u_{e}
$$

where $N^{\prime}$ is the number of atoms in the near region (inner slab, see Fig. 1), $N_{a}^{e}$ is the (total) number of atoms lying under element $e, u_{e}$ is the potential energy per atom, and $N_{e}$ is the total number of elements. Likewise, we can write the free-energy contribution $F_{q}$ to $V_{\text {eff }}$ as

$$
F_{q}=\sum_{e=1}^{N_{e}} N_{q}^{e} f_{q}^{e}
$$

where $f_{q}^{e}$ is the Helmholtz energy per atom and $N_{q}^{e}$ is the number of secondary (i.e., non-nodal) atoms under element e. From Eqs. (3.9), (3.16), and (3.17) we obtain the coarsegrained approximation 


$$
V_{\text {eff }}\left(\mathbf{r}^{N_{p}}\right)=\sum_{i=1}^{N^{\prime}} u_{i}+\sum_{e=1}^{N_{e}} N_{a}^{e} u_{e}+\sum_{e=1}^{N_{e}} N_{q}^{e} f_{q}^{e} .
$$

The expression for $u_{i}$ is given by Eq. (2.2). It remains to calculate $u_{e}$ and $f_{q}^{e}$.

Mixed local-non-local approximation. In the original QC method $^{2}$ the computation of $u_{e}$ depends on the status of element $e$ (i.e., whether $e$ is local or nonlocal, according to the criteria laid down in Sec. I B). If $e$ is local, then $u_{e}$ is taken to be equal to $u$ for the perfect (infinite) crystal at $T=0 \mathrm{~K}$ subject to the same strain as the piece of crystal under $e$. From Eq. (2.2) we have

$$
u_{e}=u_{i}=\frac{1}{2} \sum_{j \neq i} \phi\left(r_{i j}\right), \quad r_{i j}<r_{c}
$$

since all atoms of the perfect crystal are equivalent. Here the index $i$ refers to any atom of the infinite perfect crystal taken as a reference and the summation on $j$ runs over all neighbors of $i$ that lie within the cutoff sphere. On the other hand, if element $e$ is nonlocal, then $u_{e}$ is approximated by the same formula [Eq. (3.19)], but the reference atom is now taken to be the atom nearest the centroid of $e$ and the summation on $j$ to run explicitly over the neighbors of $i$ within the cutoff sphere, regardless of which elements they lie under or of whether they lie in the near region (inner slab) or not.

For a local element $f_{q}^{e}$ should, by analogy with the original QC method, be taken to be equal to $f$ for the perfect infinite crystal under the same strain as the part of the crystal under $e$. By analogy with Eq. (3.14) we have for the perfect crystal

$$
f_{q}^{e}=f=F / N=3 k_{B} T \ln \left[\hbar(\operatorname{det} \mathbf{D})^{1 / 6} / k_{B} T\right]
$$

because of the equivalence of all atoms. The dynamical matrix $\mathbf{D}$, which refers to any atom $i$ as a reference, is given by Eq. (3.15). For a nonlocal element one should use the same formula [Eq. (3.20)], taking the reference atom to be the "centroid" atom and evaluating $\mathbf{D}$ according to Eq. (3.15) by summing over neighbors explicitly.

Note that the computation of $u_{e}\left(f_{q}^{e}\right)$ for local elements is much less demanding than that for nonlocal elements. If an element is local, the reference atom "sees" only neighboring atoms that lie under itself. They belong to the same uniformly strained crystal and their positions can be calculated from a knowledge of the positions of the nodes of that element alone. In contrast, if an element is nonlocal, then the reference atom sees neighbors not only under itself but also under all neighboring elements intersected by the cutoff sphere. The positions of atoms under intersected elements of course depend on the positions of the nodes of all those elements.

All-local approximation. In a prior article, ${ }^{13}$ in which we proposed the free-energy functional method as an exact nonzero-temperature analog of the original QC method, ${ }^{2}$ we observed discontinuities in the local stress in regions of a homogeneous crystal where local and nonlocal elements are juxtaposed, or in regions where the elements are strictly nonlocal but vary in their dimensions. These discontinuities reflect so-called "ghost forces," which are spurious forces on the nodes that give rise to false minima in the free-energy functional. They are due to asymmetric contributions to $V_{\text {eff }}$ from conterminous local and nonlocal elements or from contiguous nonlocal elements that differ in their dimensions.

Although prescriptions to counteract ghost forces have been suggested, ${ }^{9,10,20}$ all of these necessitate the explicit handling of nonlocal elements, which is extraordinarily intensive computationally. We proposed an alternative remedy for the ghost-force malady: just regard all elements as if they were local, irrespective of their actual status. ${ }^{13}$ This prescription, which we call the "all-local" approximation, was successful in tests of the free-energy functional technique on our prototype: the 3D Lennard-Jones $(12,6)$ crystal. We therefore employ it here again in testing the HACG methods (see Secs. V and VI).

\section{COARSE-GRAINED THERMOMECHANICAL PROPERTIES}

Supposing that the controlled thermodynamic state variables are $\left\{T, N, L_{x}, L_{y}, L_{z}\right\}$ [see Eqs. (2.4) and (2.5)], we perform our statistical-thermodynamic analysis within the framework of the canonical ensemble, whose characteristic function, the Helmholtz energy $F\left(T, N, L_{x}, L_{y}, L_{z}\right)$, can be expressed in terms of the canonical partition function $Q$ as

$$
F=-k_{B} T \ln Q,
$$

where $Q$ is given by Eq. (3.1).

\section{A. Global stress}

From Eqs. (4.1), (2.6), and (3.4) we deduce

$$
\tau_{x x}=-\frac{k_{B} T}{L_{y} L_{z}} \frac{1}{Z_{N_{p}}}\left(\frac{\partial Z_{N_{p}}}{\partial L_{x}}\right)_{T, N, L_{y}, L_{z}}
$$

and similar expressions for the $y y$ and $z z$ components of the global stress tensor, where the configurational integral $Z_{N_{p}}$ is defined by

$$
Z_{N_{p}} \equiv \int d \mathbf{r}^{N_{p}} \exp \left[-V_{\mathrm{eff}}\left(\mathbf{r}^{N_{p}}\right) / k_{B} T\right]
$$

and $V_{\text {eff }}$ is given by Eq. (3.9). Following the standard procedure, ${ }^{23}$ we evaluate the partial derivative in Eq. (4.2), obtaining

$$
\begin{aligned}
\tau_{x x}= & -\frac{N_{p} k_{B} T}{V}+\frac{1}{V}\left\langle\sum_{i=1}^{N_{p}} \frac{\partial V_{\mathrm{eff}}}{\partial x_{i}} x_{i}\right\rangle \\
= & -\frac{N_{p} k_{B} T}{V}+\frac{1}{V}\left\langle\sum_{i=1}^{N^{\prime}} \frac{\partial V_{\mathrm{eff}}}{\partial x_{i}} x_{i}\right\rangle \\
& +\frac{1}{V}\left\langle\sum_{k=1}^{N_{n}} \frac{\partial V_{\mathrm{eff}}}{\partial X_{k}} X_{k}\right\rangle .
\end{aligned}
$$

Here $V=L_{x} L_{y} L_{z}$ stands for the volume of the system. The brackets signify an ensemble average of the enclosed dynamical quantity. In general, 


$$
\langle G\rangle \equiv \int d \mathbf{r}^{N_{p}} \rho_{B}\left(\mathbf{r}^{N_{p}}\right) G\left(\mathbf{r}^{N_{p}}\right),
$$

where the configurational distribution function is defined by

$$
\rho_{B}\left(\mathbf{r}^{N_{p}}\right) \equiv \exp \left[-V_{\text {eff }}\left(\mathbf{r}^{N_{p}}\right) / k_{B} T\right] Z_{N_{p}}^{1} .
$$

Now exploiting the relation in Eq. (2.9), we can recast the summation over nodes appearing in Eq. (4.4) generally as

$$
\sum_{k=1}^{N_{n}} \frac{\partial V_{\text {eff }}}{\partial R_{k \alpha}} R_{k \alpha}=\sum_{i=1}^{N^{\prime \prime}} \frac{\partial V_{\text {eff }}}{\partial r_{i \alpha}} r_{i \alpha},
$$

where $N^{\prime \prime}$ is the number of atoms underlying the coarsegraining mesh. ${ }^{14}$ Inserting Eq. (4.7) into Eq. (4.4), we can express the $x x$ component of the stress tensor strictly in terms of atomic coordinates,

$$
\tau_{x x}=-\frac{N_{p} k_{B} T}{V}+\frac{1}{V}\left\langle\sum_{l=1}^{N} \frac{\partial V_{\mathrm{eff}}}{\partial x_{l}} x_{l}\right\rangle .
$$

Analogous expressions obtain for the $y y$ and $z z$ components.

We turn next to the derivation of an explicit formula for the ensemble average in Eq. (4.8). From Eq. (3.18) we have

$$
\begin{aligned}
\left\langle\sum_{l=1}^{N}\right. & \left.\frac{\partial V_{\text {eff }}}{\partial r_{l \alpha}} r_{l \alpha}\right\rangle \\
= & \sum_{i=1}^{N^{\prime}}\left\langle\sum_{l=1}^{N} \frac{\partial u_{i}}{\partial r_{l \alpha}} r_{l \alpha}\right\rangle+\sum_{e=1}^{N_{e}} N_{a}^{e}\left\langle\sum_{l=1}^{N} \frac{\partial u_{e}}{\partial r_{l \alpha}} r_{l \alpha}\right\rangle \\
& +\sum_{e=1}^{N_{e}} N_{q}^{e}\left\langle\sum_{l=1}^{N} \frac{\partial f_{q}^{e}}{\partial r_{l \alpha}} r_{l \alpha}\right\rangle \\
= & \sum_{i=1}^{N^{\prime}}\left\langle W_{i \alpha}\right\rangle+\sum_{e=1}^{N_{e}} N_{a}^{e}\left\langle W_{e \alpha}\right\rangle+\sum_{e=1}^{N_{e}} N_{q}^{e}\left\langle X_{e \alpha}\right\rangle,
\end{aligned}
$$

where the second line defines for convenience the quantities $W_{i \alpha}, W_{e \alpha}$, and $X_{e \alpha}$, which are evaluated explicitly in terms of the atomic interactions in Appendix C. Combination of Eqs. (4.8) and (4.9) gives

$$
\begin{aligned}
\tau_{x x}= & -\frac{N_{p} k_{B} T}{V} \\
& +\frac{1}{V}\left[\sum_{l=1}^{N^{\prime}}\left\langle W_{i x}\right\rangle+\sum_{e=1}^{N_{e}} N_{a}^{e}\left\langle W_{e x}\right\rangle+\sum_{e=1}^{N_{e}} N_{q}^{e}\left\langle X_{e x}\right\rangle\right] .
\end{aligned}
$$

This is the $x x$ component of the "global" stress. Like formulas for the $y y$ and $z z$ components hold. Note that $\tau_{\alpha \alpha}$ refers to the whole system. The global mean stress is given by Eq. (2.7).

\section{B. Local stress}

Following previous authors, ${ }^{24,25}$ we define the local stress at an atom (more precisely, in the volume of an atom) or in an element by formally recasting the global expression in Eq. (4.10) as

$$
\begin{aligned}
\tau_{\alpha \alpha} V= & \sum_{i=1}^{N^{\prime}}\left[-\frac{k_{B} T}{V_{i}}+\frac{\left\langle W_{i \alpha}\right\rangle}{V_{i}}\right] V_{i} \\
& +\sum_{e=1}^{N_{e}}\left[-\frac{N_{n}^{e} k_{B} T}{V_{e}}+\frac{N_{a}^{e}\left\langle W_{e \alpha}\right\rangle}{V_{e}}+\frac{N_{q}^{e}\left\langle X_{e \alpha}\right\rangle}{V_{e}}\right] V_{e},
\end{aligned}
$$

where $V_{i}$ and $V_{e}$ are, respectively, the "volumes" of atom $i$ and element $e$ and $N_{n}^{e}$ is the number of nodal atoms under $e$. We identify the coefficient of $V_{i}$ or $V_{e}$ as the $\alpha \alpha$ component of the local stress tensor,

$$
\begin{aligned}
\tau_{i, \alpha \alpha} & \equiv-\frac{k_{B} T}{V_{i}}+\frac{\left\langle W_{i \alpha}\right\rangle}{V_{i}}, \\
\tau_{e, \alpha \alpha} & \equiv-\frac{N_{n}^{e} k_{B} T}{V_{e}}+\frac{N_{a}^{e}\left\langle W_{e \alpha}\right\rangle}{V_{e}}+\frac{N_{q}^{e}\left\langle X_{e \alpha}\right\rangle}{V_{e}} .
\end{aligned}
$$

Here $V_{e}$ is just the volume of a tetrahedron, which is expressible in terms of its nodal coordinates $\left(\mathbf{R}_{k}^{e}=X_{k}^{e} \mathbf{e}_{x}+Y_{k}^{e} \mathbf{e}_{y}+Z_{k}^{e} \mathbf{e}_{z}\right.$, $k=1,2,3,4)$ as

$$
V_{e}=\frac{1}{6}\left|\begin{array}{cccc}
1 & 1 & 1 & 1 \\
X_{1}^{e} & X_{2}^{e} & X_{3}^{e} & X_{4}^{e} \\
Y_{1}^{e} & Y_{2}^{e} & Y_{3}^{e} & Y_{4}^{e} \\
Z_{1}^{e} & Z_{2}^{e} & Z_{3}^{e} & Z_{4}^{e}
\end{array}\right| .
$$

The (instantaneous) volume of atom $i$ is more difficult to reckon. One possibility would be to approximate $V_{i}$ by the mean volume of atoms within a "control sphere" centered on $i .^{26}$ Another, more exacting, procedure would be to employ Voronoi tessellation, in which atom $i$ occupies a polyhedron whose faces bisect line segments connecting $i$ to its nearest neighbors. ${ }^{27}$ However, as both of these procedures are rather intensive computationally, we adopt the crude approximation that the volume assigned to every atom is fixed at $V_{i}=\rho^{-1}$, the reciprocal of the global density. By analogy with Eq. (2.7) we define the local mean stress at atom $i$ and in element $e$, respectively, by

$$
\begin{aligned}
& \bar{\tau}_{i}=\frac{1}{3} \sum_{\alpha} \tau_{i, \alpha \alpha}, \\
& \bar{\tau}_{e}=\frac{1}{3} \sum_{\alpha} \tau_{e, \alpha \alpha} .
\end{aligned}
$$

The definition of local stress that we adopt here is problematic in several respects. One is the approximation $V_{i}$ $=\rho^{-1}$, which clearly is accurate only in homogeneous regions, where every atom is subject to the same environment (e.g., homogeneously strained perfect crystal). As the degree of heterogeneity increases, the accuracy of the approximation decreases accordingly. Further the definition appears to be equivalent to what is customarily referred to as the "virial stress," questions about the interpretation of which as a true mechanical stress have been raised recently. ${ }^{28}$ Although these concerns must yet be resolved, they need not preclude our usage of the defined local stress in the present context. Our specific purpose is to determine whether the HACG methods 
generate atomic configurations of the system that are so weighted as to yield (ensemble average) local properties in agreement with the "exact" ones (i.e., those of the system treated completely at the atomistic scale). For this purpose we choose a local property, namely, the local stress as defined in Eq. (4.12), that is highly sensitive to the configurational weighting. In states of thermodynamic equilibrium all atoms of the prototype are equivalent in that they are all subject to exactly the same environment. The local mean stress $\bar{\tau}_{i}\left(\bar{\tau}_{e}\right)$ is therefore uniformly constant and equal to the global mean stress $\bar{\tau}$ throughout the system. This observation forms the basis of our quantitative assessment of the performance of the HACG techniques. Other local properties such as number density or potential-energy density might also serve our purpose, although they seem to offer less sensitive measures.

\section{STANDARD HACG COMPUTER SIMULATION}

\section{A. General procedure}

In the limit $T=0 \mathrm{~K}$ we employ the conjugate gradient technique ${ }^{29}$ to minimize the (effective) potential energy. The initial configuration is "random" [i.e., it is generated from the reference configuration (see Fig. 1) by giving each atom (node) a random displacement within a small cube centered on the atom (node)].

In case $T>0$ the Helmholtz energy is effectively minimized by means of the Metropolis Monte Carlo procedure. ${ }^{15}$ The initial configuration is taken to be the "randomized" final (minimum potential) configuration obtained at $T=0 \mathrm{~K}$. A MC cycle consists of $N^{\prime}$ sequential random trial displacements of atoms in the inner slab (near region) followed by $N_{n}$ sequential random displacements of nodes in the outer slabs (coarse-grained far regions). The effective potential energy governing acceptance of trial moves is given by Eq. (3.18). Separate maximum displacements $\left(d r_{\max }\right)$ for atomic and nodal moves are periodically adjusted so that about $50 \%$ of attempted moves are accepted. A typical MC run consists of $10^{5}$ cycles. The first $2 \times 10^{4}$ "equilibration" cycles are discarded and the remaining ones are used to compute ensemble averages. For the latter purpose the MC sequence is sampled every cycle.

To ascertain the reliability of the HACG techniques, we simulate the same thermodynamic states of the system in the fully atomistic limit and take the "atomistic" properties to be exact.

All computations are performed, and all results are stated, in dimensionless units based on the parameters of the Lennard-Jones potential (see Appendix B).

\section{B. Fully nonlocal treatment at $T=0 \mathrm{~K}$}

We test the standard HACG methodology ${ }^{17}$ by applying it first to the several prototypal systems whose parameters are listed in Table I. Since the cutoff radius $r_{c}(=2.5)$ is greater than one-half of the smaller $(x, y)$ overall dimension of the elements, $3 a / 2(\approx 2.25)$, all elements are strictly nonlocal and $u_{e}$ and $f_{q}^{e}$ are computed according to the mixed local-non-local formulation described in Sec. III C. Figure 2 displays a plot of local mean stress versus $z$ for system I at
TABLE I. Parameters of system pictured in Fig. 1. In all cases the following are fixed, unless otherwise indicated: $L_{x}=L_{y}=6 a, n_{x}=n_{y}=6, n_{z}^{\prime}=6, k_{z}=3$, $N^{\prime}=792$, and $\rho=1.190(a=1.498)$.

\begin{tabular}{lccc}
\hline \hline System & I & II & III \\
\hline$m_{x}\left(=m^{y}\right)$ & 3 & 2 & 3 \\
$k_{x}\left(=k_{y}\right)$ & 2 & 3 & 2 \\
$m_{z 1}$ & 8 & 8 & 8 \\
$m_{z 2^{\prime}}$ & 4 & 4 & 4 \\
$m_{z 3}$ & 3 & 3 & 9 \\
$n_{z}^{\prime \prime}$ & 15 & 15 & 21 \\
$N_{e}$ & 144 & 324 & 144 \\
$N_{n}$ & 28 & 63 & 28 \\
$N_{p}$ & 820 & 855 & 820 \\
$N$ & 5184 & 5184 & 6912 \\
\hline \hline
\end{tabular}

$T=0$. The discrete points correspond to values of the local mean stress $\left[\bar{\tau}_{i}\right.$ or $\bar{\tau}_{e}$, see Eq. (4.14)] at atoms or in elements. These values are projected onto the $z$ axis at the position of the atom or of the centroid of the element. The plot may therefore be multivalued in some locations because of the variation of the stress in the $x$ and $y$ directions. One would expect the plot to be a horizontal line whose intercept coincides with the value of the global mean stress (indicated by the horizontal dashed line in Fig. 2), since the stress in a perfect crystal in a state of thermodynamic equilibrium should be uniform. We observe, however, that $\bar{\tau}_{i}\left(\bar{\tau}_{e}\right)$ is uniformly equal to the global value $\bar{\tau}$ only in the far regions of the crystal well removed from the near-far interfaces, in the vicinity of which strong discontinuities appear. Note that the symmetry of the plot about the midplane $\left(z / a=L_{z} / 2 a=18\right)$ suggests that the numerical method is properly implemented, even though use of Eq. (3.18) somehow gives rise to the observed discontinuities in $\bar{\tau}_{i}\left(\bar{\tau}_{e}\right)$.

Although $\bar{\tau}_{e}$ is very nearly equal to $\bar{\tau}$ in the two outermost subprisms (top or bottom slab), it exhibits discontinuities at the boundaries between the second and third subprisms and between the third subprism and the inner slab (atomistic near region). These discontinuities are traceable to

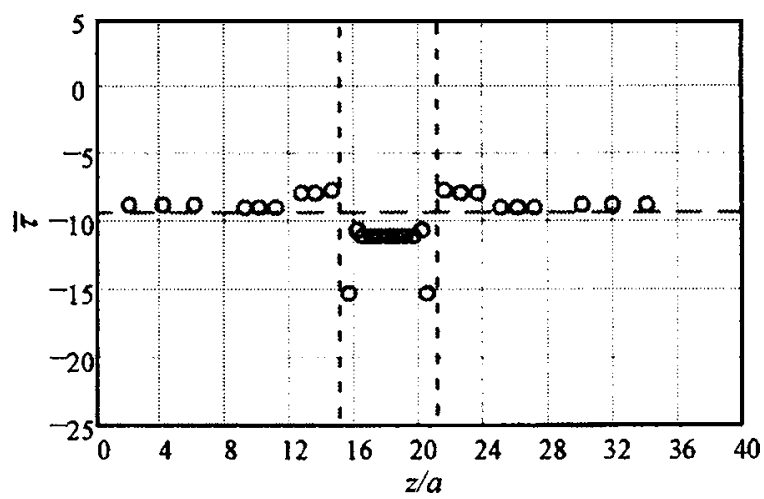

FIG. 2. Local mean stress $\left(\bar{\tau} \equiv \bar{\tau}_{e}, \bar{\tau}_{t}\right)[$ Eq. (4.14)] vs $z / a$ for system I (see Table I) at $T=0$, computed treating elements according to their true status (nonlocal). The vertical dotted lines indicate positions of planes $z=n_{z}^{\prime \prime} a$ and $z=\left(n_{z}^{\prime \prime}+n_{z}^{\prime}\right) a$ of interfaces between near and far regions. In far regions $\left(z<n_{z}^{\prime \prime} a, z>\left(n_{z}^{\prime \prime}+n_{z}^{\prime}\right) a\right)$ discrete points $\left(\bar{\tau}_{e}\right)$ plotted at positions of centroids of elements; in near region $\left(n_{z}^{\prime \prime} a<z<\left(n_{z}^{\prime \prime}+n_{z}^{\prime}\right) a\right)$ discrete points $\left(\bar{\tau}_{i}\right)$ plotted at positions of atoms. The horizontal dashed line indicates "exact" global mean stress computed in fully atomistic limit. 


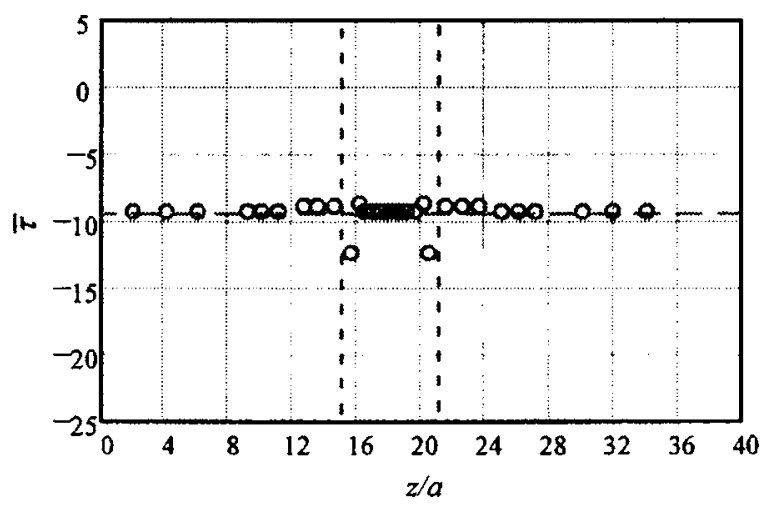

FIG. 3. Same as Fig. 2, except that all elements treated as local.

ghost forces (see discussions in Secs. III C and IVD), which are due to asymmetric contributions to $U\left(\mathbf{r}^{N_{p}}, \mathbf{0}\right)$ of two types: (1) from conterminous nonlocal elements of different dimensions at the boundary between the second and third subprisms and (2) from elements in the third subprism and atoms in the near region.

\section{All-local approximation}

According to the discussion in Sec. III C, we were able, in the context of the free-energy functional technique, to eliminate the impact of ghost forces at nodes shared by local and nonlocal elements, or by nonequivalent nonlocal elements, simply by treating all elements as if they were local, regardless of their true status. The result of our attempt to do so in the present context of the standard HACG simulation is shown in Fig. 3, where we plot local mean stress versus $z$ for the same system (I) to which Fig. 2 pertains, except that we employ the "all-local" approximation to $V_{\text {eff. }}$ Although the local stress still exhibits discontinuities in elements bordering the near-far interfaces and at atoms in the two layers closest to the interfaces, their magnitude is much reduced. We therefore utilize the all-local approximation henceforth.

\section{Origin of ghost forces}

The all-local approximation clearly does not eliminate ghost forces, as the plot in Fig. 4 indicates. The essential reason for its failure can be seen by explicitly calculating the

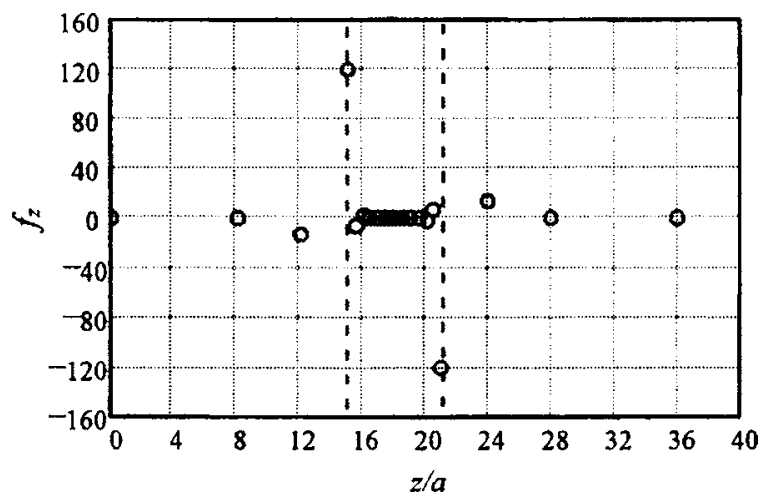

FIG. 4. $z$ component of "ghost force" on node (atom) vs $z / a$ for system I (see Table I), in which atoms are in configuration of perfect crystal at $T$ $=0$. The vertical dotted lines define regions (see Fig. 2). Discrete points plotted at positions of atoms or nodes. ghost force on an atom at a near-far interface in the perfect crystal at $T=0$. This is the true state of minimum potential energy; the forces on all atoms, which are in equivalent environments, should be zero. However, consider an atom $(l)$ sufficiently close to an interface that it interacts with atoms lying under the contiguous (interfacial) elements, as illustrated in Fig. 1. From Eqs. (3.18) and (2.2) we compute the force acting on $l$,

$$
\mathbf{f}_{l}=-\nabla_{\mathbf{r}_{l}} V_{\text {eff }}=\frac{1}{2}\left[\sum_{k \neq l} \phi^{\prime}\left(r_{k l}\right) \mathbf{r}_{k l} / r_{k l}+\sum_{k \neq l}^{N^{\prime}} \phi^{\prime}\left(r_{k l}\right) \mathbf{r}_{k l} / \mathbf{r}_{k l}\right] .
$$

Here the prime on $\phi^{\prime}$ denotes the derivative with respect to the argument. Note that the first summation on the right side of Eq. (5.1) runs over all atoms $k$ lying within the cutoff sphere, whereas the second runs over only atoms within the cutoff sphere that lie in the near region (inner slab). Were it not for this asymmetry, we could combine the sums to obtain

$$
\left(\mathbf{f}_{l}\right)_{\text {exact }}=\sum_{k \neq l} \phi^{\prime}\left(r_{k l}\right) \mathbf{r}_{k l} / \mathbf{r}_{k l},
$$

which is the expression for the exact force on $l$. On account of the symmetry of the perfect crystal, the terms in the sum in Eq. (5.2) cancel in pairs to yield $\left(\mathbf{f}_{l}\right)_{\text {exact }}=\mathbf{0}$. Because of the absence of counterbalancing terms in the second sum in Eq. (5.1), $\mathbf{f}_{l}$ does not vanish. It is indeed the spurious ghost force that arises on account of the noted asymmetry at the interface. In essence, one-half of the potential energy of interaction between the atom $l$ and its neighbors under the contiguous elements is subsumed into $u_{e}$, which does not change as $l$ moves. Obviously, a near-region atom sufficiently distant from the interface that it interacts only with other near-region atoms "feels" no ghost force.

When an interfacial node is moved, the (equilibrium positions of) atoms underlying all elements that share the node move in concert. However, the atoms in the contiguous near region remain stationary. This asymmetry leads to a net (ghost) force on the node. When a node located sufficiently far away from the interface is moved, then the distortions of the (assumed) local elements that share the node compensate one another so as to yield no net force on the node.

As a consequence of ghost forces the configuration determined by minimizing the approximate $V_{\text {eff }}$ at $T=0$ is not that of the perfect crystal, but rather is distorted. The distortion is of course reflected in the discontinuities in the local stress.

\section{SELF-CONSISTENT-FIELD HACG METHODOLGY}

One possible recipe to neutralize ghost forces at $T=0$ is to apply a constant external "dead load" $\mathbf{f}_{i}^{\text {ex }}$ to each node and atom such that minimization of a modified effective potential energy

$$
V_{\mathrm{eff}}^{\prime}=V_{\mathrm{eff}}+\sum_{i=1}^{N_{p}} \mathbf{f}_{i}^{\mathrm{ex}} \cdot \mathbf{u}_{i}
$$

yields the correct configuration. In Eq. (6.1) $\mathbf{u}_{i}$ stands for the displacement of the atom or node from its reference position 
in the perfect crystal at $T=0$. The condition for a minimum of $V_{\mathrm{eff}}^{\prime}$ is

$$
\mathbf{f}_{l}^{\prime}=-\nabla_{\mathbf{r}_{l}} V_{\mathrm{eff}}^{\prime}=-\nabla_{\mathbf{r}_{l}} V_{\mathrm{eff}}-\mathbf{f}_{l}^{\mathrm{ex}}=\mathbf{0}, \quad l=1,2, \ldots, N_{p} .
$$

Therefore, the dead load on atom (node) $l$ should be $\mathbf{f}_{l}^{\mathrm{ex}}=-\nabla_{\mathbf{r}_{l}} V_{\mathrm{eff}}=\mathbf{f}_{l}$, which is just the ghost force on $l$. Now minimization of $V_{\text {eff }}^{\prime}$ leads to the correct configuration at $T$ $=0$ and consequently to a uniform local mean stress.

It is unfortunate that one does not in general know $a$ priori the correct minimum $(T=0)$ configuration of the system. Moreover, our attempts to extend this "dead-load" scheme to nonzero temperature, using the ghost forces determined at $T=0$ as an approximation, failed. Many additional attempts to palliate the spoiling effects of ghost forces led us eventually to what we dub as the "self-consistent-field" (SCF) HACG method.

In the SCF-HACG Monte Carlo procedure one MC cycle comprises two stages. In stage 1 the $N_{n}$ nodes of the coarse-grained far regions are held fixed while the $N^{\prime}$ nearregion atoms of the inner slab are sequentially subjected to random displacements. The potential energy controlling acceptance of trial displacements is taken to be

$$
U_{1}=\sum_{i=1}^{N^{\prime}} u_{i}+\sum_{j=1}^{N^{\prime \prime}} u_{i}
$$

where $u_{i}$ is given by Eq. (2.2). The second summation in Eq. (6.3) runs over only the $N^{\prime \prime}$ far-region atoms that interact with near-region atoms (i.e., those far-region atoms $i$ for which $r_{i j}<r_{c}$, where $j$ refers to a near-region atom). The change in the potential energy associated with a trial movement of atom $i$ is

$$
\Delta U_{1}=\Delta\left[\sum_{j \neq i} \phi\left(r_{i j}\right)\right], \quad r_{i j}<r_{c} .
$$

Note that this expression, which follows from Eqs. (2.2) and (6.3), is identical to the one obtained from the original potential energy [Eq. (2.1)]. That is, $\Delta U_{1}$ is just the change in the potential energy of atom $i$ displaced in the field of all other atoms $j$, which remain stationary. In stage 2 the atoms in the near region are kept fixed in the final configuration of stage 1 while the $N_{n}$ nodes are given random trial displacements sequentially. We assume the potential energy governing acceptance of nodal movements is

$$
U_{2}=\sum_{e} N_{a}^{e} u_{e}+\sum_{e} N_{q}^{e} f_{q}^{e}-\sum_{e}^{\prime} \sum_{i}^{\prime} \mathbf{f}_{i}^{e} \cdot \mathbf{d}_{i}^{e},
$$

where $\mathbf{f}_{i}^{e}$ is the "external" force on atom $i$ under element $e$ due to near-region atoms and $\mathbf{d}_{i}^{e}$ is the displacement of $i$ from its reference position at the start of stage 2 . The force is given by the exact expression

$$
\mathbf{f}_{i}^{e}=-\nabla_{\mathbf{r}_{i}} U=-\sum_{j \neq l} \phi^{\prime}\left(r_{i j}\right) \mathbf{r}_{i j} / r_{i j}, \quad r_{i j}<r_{c},
$$

where the summation of $j$ runs over only those atoms that lie in the near region within the cutoff sphere. The primes on the summation symbols in Eq. (6.5) serve to emphasize the restriction that atom $i$ lie within a distance $r_{c}$ from the closest
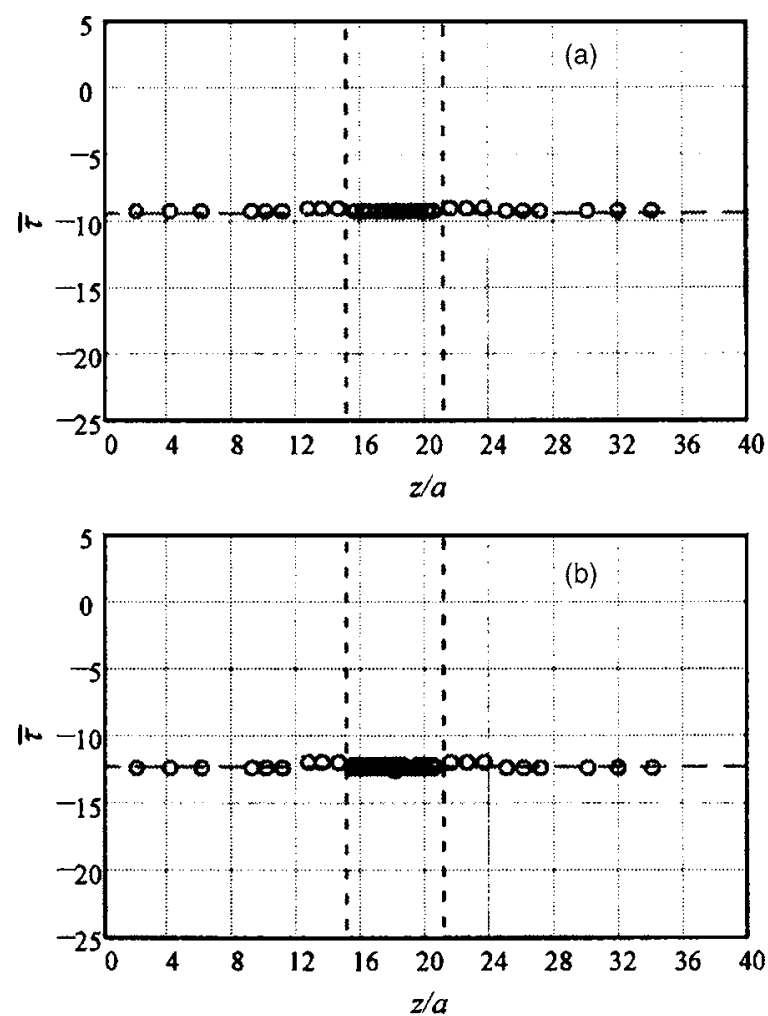

FIG. 5. Same as Fig. 2, except system simulated by SCF HACG scheme. (a) $T=0$; (b) $T=0.3$.

near-region atom. The $\mathbf{f}_{i}^{e}$ are kept fixed (i.e., "dead loads") at the initial values throughout stage 2 . This approximation, which amounts to the Taylor series expansion of the exact potential energy (as a function of the coordinates of the underlying atom) truncated at first order, should be reasonable in light of the small displacements of the nodes compared with the displacements of atoms in stage 1.

The change in the potential energy accompanying the trial displacement of a node can be expressed as

$$
\Delta U_{2}=\sum_{e}{ }^{\prime} N_{a}^{e} \Delta u_{e}+\sum_{e}{ }^{\prime} N_{q}^{e} \Delta f_{q}^{e}-\sum_{e}^{\prime} \sum_{i}^{\prime} \mathbf{f}_{i}^{e} \cdot \Delta \mathbf{d}_{i}^{e},
$$

where the primes on the first two summations on $e$ signify the restriction to elements sharing the moved node and $\Delta x$ denotes the change in the quantity $x$. Note that the restriction on the sums to elements that share the moved node reflects the "all-local" approximation. The first two terms of Eq. (6.7) give the change in the "internal" (effective) potential energy of the coarse-grained far regions; the third term represents the change in the potential energy due to the external field arising from the near region.

Results of the SCF-HACG simulation of system I (see Table I) at $T=0$ are shown in Fig. 5(a). Comparison of Fig. 3 with Fig. 5(a) reveals that the SCF method essentially eliminates the discontinuities in local mean stress at near-region atoms close to the near-far interfaces. On the other hand, a small discontinuity in the local stress remains in elements that border the interface. Figure 5(b), which displays a plot of local mean stress versus $z$ for the same system (I) at $T$ $=0.30$, shows that the SCF technique works comparably well at nonzero temperature, although there is now evidence 

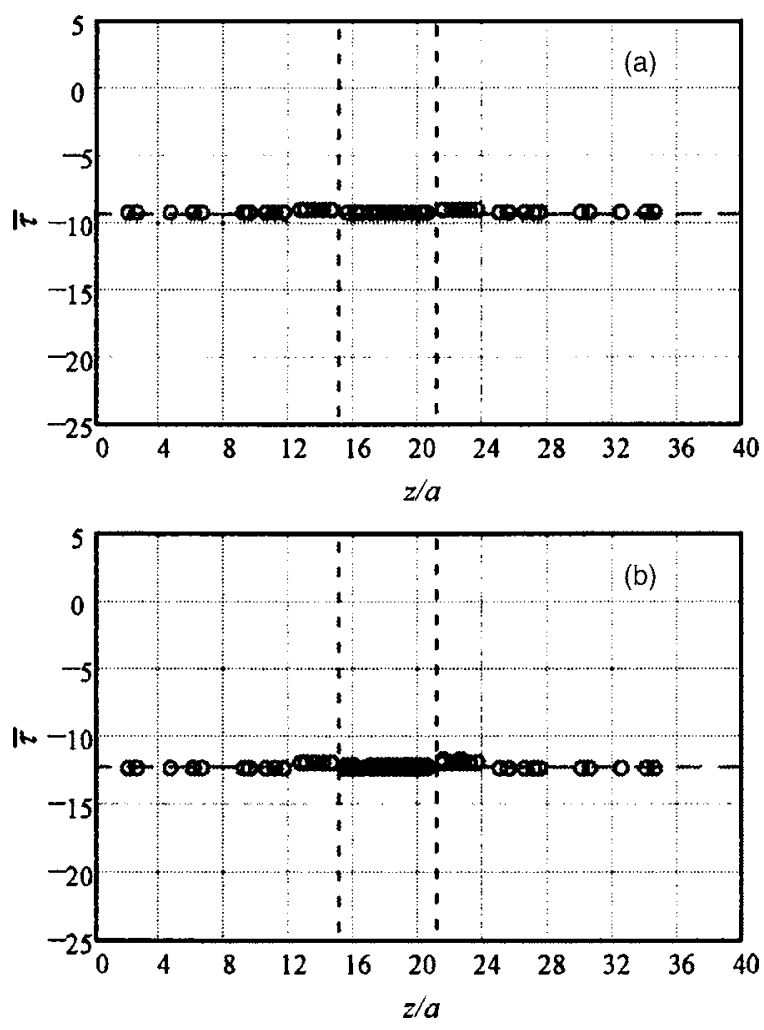

FIG. 6. Same as Fig. 5, except for system II (see Table I).

(smearing of discrete points due to variation of $\bar{\tau}_{i}$ in the $x$ and $y$ directions) of greater nonuniformity of local stress in the near region. We note also that the slight discrepancy between coarse-grained and exact values is due to the limitations of the harmonic approximation.

The effect of finer partitioning of the far regions in the $x$ and $y$ dimensions is shown in Fig. 6, where we plot $\bar{\tau}_{i}\left(\bar{\tau}_{e}\right)$ vs $z$ for system II defined in Table I. Comparing Fig. 6 with Fig. 5 , we observe that the results are essentially unchanged. The considerable additional expense incurred by use of a finer coarse-graining mesh does not yield a significant return in accuracy. In contrast, coarsening the mesh in the $z$ direction at the near-far interfaces leads to a distinct gain in accuracy for less computational effort, as shown in Fig. 7, which refers to system III (see Table I). We note that the main improvement appears in $\bar{\tau}_{e}$ for the elements that border the interfaces. That these elements are more nearly local in the $z$ dimension, which is indicated by the greater distances of the centroids from the interfaces, may rationalize the improvement. It is encouraging to know that one may be able to employ truly local elements at near-far interfaces, as long as the integrity of the near region in the vicinity of the interfaces is maintained during the process of interest.

\section{APPLICATION OF THE SCF-HACG METHOD TO BICRYSTAL}

To this juncture we have presented only the results of tests of the new SCF-HACG scheme on an idealized system whose thermodynamic states and their properties are known. Our ultimate aim is to utilize the method in studies of more complex systems of practical import, such as polycrystalline
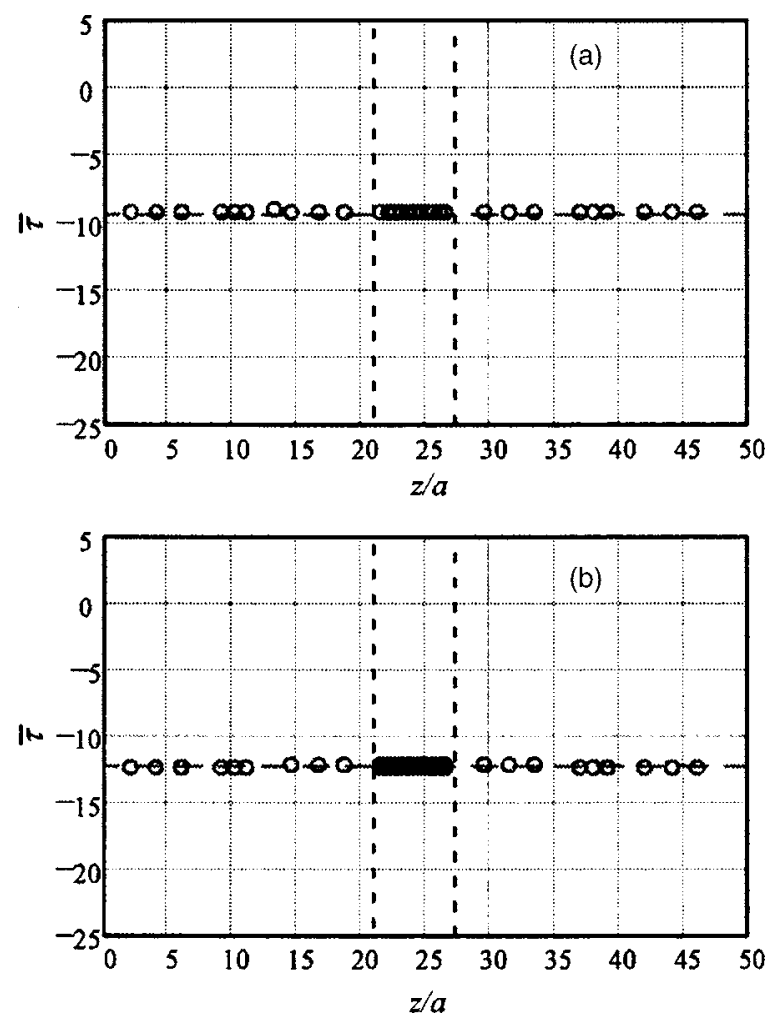

FIG. 7. Same as Fig. 5, except for system III (see Table I).

films or fibers, which are in general not in states of thermodynamic equilibrium. To get some idea of the viability of the SCF-HACG MC technique in a practical setting, we apply it here to the antisymmetrically tilted bicrystal, which is frequently employed as a model for investigation of the properties of grain boundaries. ${ }^{30}$

Our prototypal bicrystal, which is pictured in Fig. 8, is constructed as follows. A perfect Lennard-Jones $(12,6)$ crystal is cleaved along the (201) plane and a second such crystal is cleaved along the (201) plane. "One-half" of each crystal is discarded, the remaining halves are rotated relative to each other about the $y$ axis ([010] direction) by the misorientation angle $\theta$, and the (201) face of one crystal is superposed on the $(20 \overline{1})$ face of the other. The interface is oriented parallel with the $x-y$ plane and the irregular chunk is trimmed to create the prism shown in Fig. 8. The far regions are coarse grained symmetrically about the interface in a manner similar to that described in Sec. II for the single crystal. Periodic boundary conditions are imposed in all dimensions.

Plots of $\bar{\tau}_{i}\left(\bar{\tau}_{e}\right)$ vs $z$ are displayed in Fig. 9. (We note in passing that the macroscopic strain due to the bonding of the two crystals is uniaxial in the $z$ direction.) As Fig. 9 indicates, not only do relatively small residual discontinuities remain at the near-far interfaces, as they do in the single crystal (see Figs. 5-7), but strong oscillations in local stress confined close to the center of the near region also now appear. These sharp variations reflect the truly heterogeneous character of the grain boundary. Note that the scale of the abscissa in Fig. 9 is expanded so that the detailed structure of the local stress in the grain boundary can be discerned. In the homogeneous far-region ranges $(0<z<14)$ and $(26<z$ 


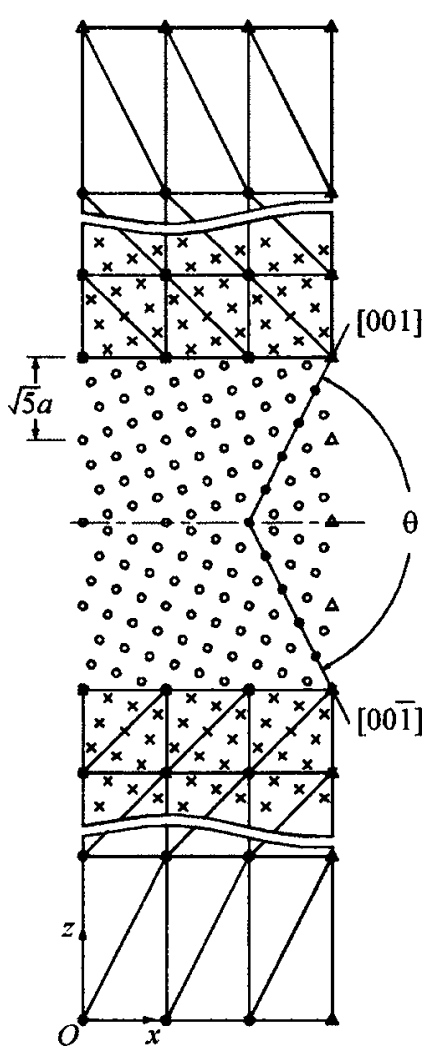

FIG. 8. Side view (along positive $y$ axis) of Lennard-Jones $(12,6)$ bicrystal with misorientation angle $\theta=126^{\circ} 52^{\prime}$. Only top layer of atoms shown in initial configuration. The open circles represent atoms in near region, crosses atoms in far regions, and filled circles nodes. The open triangles on top and right boundaries stand for periodic images. Dimensions in $x$ and $z$ directions given in units of $\sqrt{5} a$ and in $y$ direction in units of $a: L_{x}$ $=3(\sqrt{5} a) ; L_{y}=6(a) ; L_{z}=18(\sqrt{5} a) ; k_{x}=3, m_{x}=1 ; k_{y}=3, m_{y}=2 ; k_{z}=3, m_{z 1}=4$, $m_{z 2}=2, m_{z 3}=1 ; n_{z}^{\prime}=4 ; n_{z}^{\prime \prime}=7$; and $n_{z}=18$.

$<40$ ), which are not visible in the plots, $\bar{\tau}_{e}$ is constant and equal to $\bar{\tau}_{i}$ in the homogeneous near-region ranges $(16<z$ $<18)$ and $(22<z<24)$. Moreover, $\bar{\tau}_{i}\left(\bar{\tau}_{e}\right)$ in these regions is very close to, but slightly higher (more negative) than, $\bar{\tau}$ for the single crystal from which the bicrystal is constructed. We ascribe this to a slight compression of those regions upon relaxation of the strained interface (where some atoms are initially crowded very close together) toward a state of "local" equilibrium.

Care should be taken in the interpretation of the plots in Fig. 9. In ranges of $z$ sufficiently removed from the grain boundary, where the atoms are in essentially equivalent environments and the atomic volume is constant, $\bar{\tau}_{i}$ is constant and equal to the global mean stress $\bar{\tau}$. However, in the grain boundary, where local density varies strongly and the atoms are subject to disparate local surroundings, the implicit assumption of a constant atomic volume is obviously wrong. Therefore the numerical values of $\bar{\tau}_{i}$ in the grain boundary should be regarded with caution. Consider, however, the bicrystal at $T=0$ and compute the "average normal stress" at a cross section parallel with the $x-y$ plane, which we define as the $z$ component of the total force on the atoms on one side of the plane (of the cross section) due to atoms on the other side, divided by the cross-sectional area. If the bicrystal is in a mechanically stable (or metastable, see discussion below)
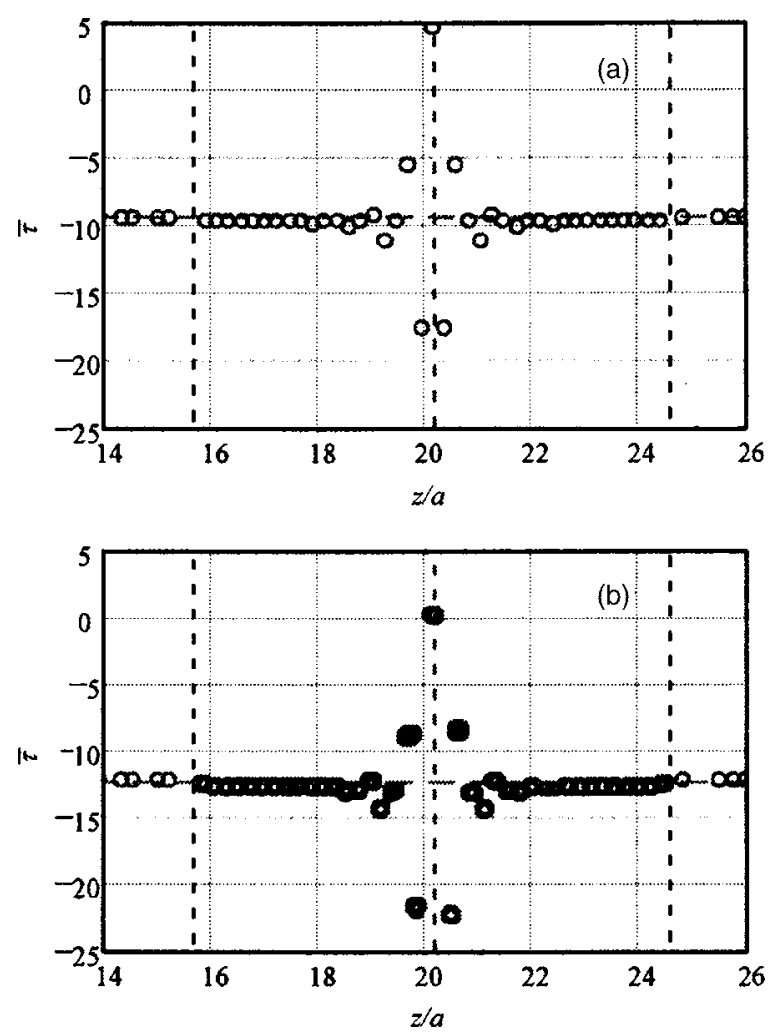

FIG. 9. Local mean stress $\left(\bar{\tau} \equiv \bar{\tau}_{e}, \bar{\tau}_{i}\right)$ vs $z$ for bicrystal described in Fig. 8 . (a) $T=0$; (b) $T=0.3$. The vertical dotted line in center represents plane in which (201) and (201) faces initially superposed. The horizontal line pertains to single crystal at same density.

state, then the magnitude of the average normal stress at any cross section should be the same. We have computed the defined average normal stress, finding it to be sensibly constant as the $z$ coordinate of the cross section traverses the grain boundary. We therefore conclude that the SCF-HACG method describes a physically realistic state of the bicrystal. [We note in passing that our definition of "average normal stress" can be viewed as the atomistic analog of the (mean) Cauchy stress defined at the continuum level. ${ }^{28}$ ]

We emphasize that the system cannot be in a state of true equilibrium, that is, in a state of minimum Helmholtz energy at the given fixed density and temperature, since this state corresponds to the original perfect single crystal. The state of the bicrystal is necessarily metastable. On the time scale of our observation, determined in essence by the number of MC cycles, as well as the nature of the allowed trial displacements (i.e., short, single-atom, or -node displacements), the system is frustrated in reaching the true equilibrium state. Nevertheless, the "frustrating" MC procedure leads to a state of local equilibrium that resembles the actual metastable state that would be observed on the experimental time scale. Our objective is, of course, to mimic this latter state.

\section{CONCLUDING REMARKS}

The self-consistent-field hybrid atomistic-coarse-grained (SCF-HACG) technique presented here allows the treatment of multiscale thermomechanical processes in heterogeneous systems that cannot be handled by the original quasicontinuum method. The new hybrid scheme has three salient 
features. (1) Only the portion of the system whose structural integrity is preserved during the process is coarse grained; the remainder is described strictly at the atomic scale. This feature conveniently permits the treatment of processes (e.g., thin-film lubrication) involving fluid-solid interfaces. (2) The thermal motion of non-nodal atoms underlying the coarsegraining mesh is properly accounted for by an effective thermodynamic state-dependent potential energy. (3) The use of a two-stage Monte Carlo cycle, in which the potential energy controlling the acceptance of trial movements depends on the stage, mitigates the impact of "ghost forces."

The new SCF-HACG technique was first tested on an ideal system whose properties can be computed by independent methods: a pure Lennard-Jones $(12,6)$ crystal. The crystal is partitioned into three slabs such that two thick outer slabs sandwich a thinner inner slab. The outer slabs (far regions) are coarse grained; the inner one (near region) is treated explicitly at the atomic scale. The presence of nearfar interfaces engenders artificial heterogeneities in the dimension normal to the interfaces. These heterogeneities are manifest in the local stress. If we employ the standard HACG procedure (see Sec. V) to simulate the crystal, we find that the local stress exhibits discontinuities near the interfaces. We note, however, that in regions sufficiently far removed from the interfaces the local stress is uniform, as it should be in the crystal in thermodynamic equilibrium. The value of the local stress also agrees with the "exact" global stress for the given thermodynamic state. In other words, while the standard HACG method functions well in regions far enough from the interfaces, it breaks down in regions close to the interfaces.

This breakdown is traceable to ghost forces on atoms (nodes) close to the near-far interfaces, which are due to errors in the forces on atoms (nodes) that arise from the approximate form of the coarse-grained effective potential energy. Investigation of various approaches to the palliation of ghost forces led us eventually to the most successful one: the SCF-HACG method. Use of the exact form of the potential energy [Eq. (2.1)] to compute $\Delta U_{1}$ in stage 1 and $\mathbf{f}_{i}$ in stage 2 effectively eliminates the imbalance of forces at the near-far interfaces that results from direct use of the coarsegrained form [Eq. (3.18)] in the standard HACG MC procedure. The two approximations invoked in stage 2, namely, the "all-local" approximation to $u_{e}$ and the constancy of the "external" forces $\mathbf{f}_{i}$, are presumably responsible for the small residual errors.

Application of the SCF-HACG method to a truly heterogeneous system, namely, the Lennard-Jones bicrystal, gives results in conformity with one's expectations. Even though small residual nonphysical discontinuities remain in the vicinity of the near-far interfaces, the truly heterogeneous grain boundary, which is entirely contained within the atomistic near region, seems to be properly characterized. On one hand, the sharp variation of the local mean stress mirrors the heterogeneity of the grain boundary. On the other hand, the constancy of the "average normal stress" throughout the grain boundary reflects the stability of the bicrystal.

\section{ACKNOWLEDGMENTS}

The authors thank the National Science Foundation (DMII/NIRT-0210850 and CHE/ITR-0427746), the U.S. Department of Energy (DE-FG0204ER 46146), the U.S. Army Research Office (DAAD19-00-1-0161), the Nebraska Research Initiative, and the University of Nebraska-Lincoln Research Computing Facility for support of the work reported here.

\section{APPENDIX A: ANALYTICAL EXPRESSION FOR $\partial r_{i j} / \partial r_{l \alpha}$}

The derivations presented in appendixes $\mathrm{B}$ and $\mathrm{C}$ call for an explicit expression for the partial derivative $\partial r_{i j} / \partial r_{l \alpha}$, where $r_{l \alpha}$ is the $\alpha$ Cartesian component of the position of atom $l$ and $r_{i j}=\left|\mathbf{r}_{i}-\mathbf{r}_{j}\right|$. We start with the relation

$$
r_{i j}^{2}=\sum_{\gamma} r_{i j \gamma}^{2}
$$

where

$$
r_{i j \gamma}=r_{i \gamma}-r_{j \gamma} .
$$

The partial derivative of Eq. (A1) yields

$$
\frac{\partial r_{i j}}{\partial r_{l \alpha}}=r_{i j}^{-1} \sum_{\gamma} r_{i j \gamma} \frac{\partial r_{i j \gamma}}{\partial r_{l \alpha}} .
$$

From Eq. (A2) we have

$$
\frac{\partial r_{i j \gamma}}{\partial r_{l \alpha}}=\frac{\partial r_{i \gamma}}{\partial r_{l \alpha}}-\frac{\partial r_{j \gamma}}{\partial r_{l \alpha}}=\left(\delta_{i l}-\delta_{j l}\right) \delta_{\alpha \gamma},
$$

where $\delta$ represents the Kronecker delta. Substituting Eq. (A4) into Eq. (A3) gives

$$
\frac{\partial r_{i j}}{\partial r_{l \alpha}}=r_{i j}^{-1} r_{i j \alpha}\left(\delta_{i l}-\delta_{j l}\right) .
$$

\section{APPENDIX B: ANALYTICAL EXPRESSION FOR $D_{\alpha \beta}$}

To obtain a closed expression for the dynamical matrix elements $D_{\alpha \beta}$, we begin with Eq. (3.15)

$$
D_{\alpha \beta}=m^{-1} \sum_{j \neq i} \frac{\partial}{\partial r_{i \alpha}} \frac{\partial \phi\left(r_{i j}\right)}{\partial r_{i \beta}},
$$

where it is implicit that the expression is to be evaluated finally at the equilibrium configuration. The first partial derivative can be written as

$$
\frac{\partial \phi}{\partial r_{i \beta}}=\phi^{\prime}\left(r_{i j}\right) \frac{\partial r_{i j}}{\partial r_{i \beta}}=\phi^{\prime}\left(r_{i j}\right) r_{i j}^{-1} r_{i j \beta},
$$

where the prime means the total derivative with respect to the argument $\left(r_{i j}\right)$ and the second line depends on Eq. (A5). Inserting Eq. (B2) into Eq. (B1), we obtain 


$$
\begin{aligned}
D_{\alpha \beta}= & m^{-1} \sum_{j \neq i} \frac{\partial}{\partial r_{i \alpha}}\left[\phi^{\prime}\left(r_{i j}\right) r_{i j}^{-1} r_{i j \beta}\right] \\
= & m^{-1} \sum_{j \neq i}\left\{\left[\phi^{\prime \prime}\left(r_{i j}\right) r_{i j}^{-2}-\phi^{\prime}\left(r_{i j}\right) r_{i j}^{-3}\right] r_{i j \alpha} r_{i j \beta}\right. \\
& \left.+\delta_{\alpha \beta} \phi^{\prime}\left(r_{i j}\right) r_{i j}^{-1}\right\} .
\end{aligned}
$$

We now use Eqs. (2.1) and (2.2) to calculate expressions for the required derivatives of $\phi$ and substitute them into Eq. (B3) to get

$$
D_{\alpha \beta}=24 \varepsilon \sigma^{-2} m^{-1} \sum_{j \neq i}\left(a_{i j}^{*} r_{i j \alpha}^{*} r_{i j \beta}^{*}-b_{i j}^{*} \delta_{\alpha \beta}\right),
$$

where

$$
\begin{aligned}
a_{i j}^{*} & \equiv 28 r_{i j}^{*-16}-8 r_{i j}^{*-10}, \\
b_{i j}^{*} & \equiv 2 r_{i j}^{*-14}-r_{i j}^{*-8},
\end{aligned}
$$

and

$$
r_{i j}^{*} \equiv r_{i j} / \sigma .
$$

We perform the computer simulations, and state the results, in reduced (dimensionless) units based on the LennardJones $(12,6)$ interatomic potential. Distance is given in units of $\sigma$, mass in units of the mass $m$ of the atom, energy in units of $\varepsilon$, time in units of $\left(m \sigma^{2} \varepsilon^{-1}\right)$, and temperature in units of $\varepsilon k_{B}^{-1}$. An asterisk attached to a symbol signifies a reduced dimensionless quantity. From Eq. (B4) we see that in dimensionless units the dynamical matrix elements are

$$
D_{\alpha \beta}^{*}=24 \sum_{j \neq i}\left(a_{i j}^{*} r_{i j \alpha}^{*} r_{i j \beta}^{*}-b_{i j}^{*} \delta_{\alpha \beta}\right) .
$$

Now casting Eq. (3.20) in dimensionless units, we have

$$
f_{q}^{e^{*}}=f_{q}^{e} / \varepsilon=3 T^{*} \ln \left[\hbar^{*}(\operatorname{det} \mathbf{D} *)^{1 / 6} / T^{*}\right],
$$

where

$$
\hbar^{*} \equiv \hbar\left(\varepsilon m \sigma^{2}\right)^{-1 / 2}
$$

is the reduced (modified) Planck constant. Since $\hbar$ is a fixed constant, we need to adopt a specific material as reference in order to evaluate $\hbar^{*}$. We arbitrarily select Ar, for which $\sigma$ $=3.405 \times 10^{-10} \mathrm{~m}, \quad \varepsilon=1.656 \times 10^{-21} \mathrm{~J}, \quad$ and $\quad m=6.633$ $\times 10^{-26} \mathrm{~kg}$. Then $\hbar^{*}=0.02956$.

\section{APPENDIX C: EXPLICIT EXPRESSIONS FOR $W_{i \alpha}, W_{e \alpha}, X_{e \alpha}$}

Recalling that $u_{i}$ for a near-region atom is expressed by Eq. (3.17), we have from the definition of $W_{i \alpha}$ given by Eq. (4.9)

$$
\begin{aligned}
W_{i \alpha} & \equiv \sum_{l=1}^{N} \frac{\partial u_{i}}{\partial r_{l \alpha}} r_{l \alpha} \\
& =\frac{1}{2} \sum_{j \neq i} \sum_{l=1}^{N} \frac{\partial \phi\left(r_{i j}\right)}{\partial r_{l \alpha}} r_{l \alpha} \\
& =\frac{1}{2} \sum_{j \neq i} \phi^{\prime}\left(r_{i j}\right) \sum_{l=1}^{N} \frac{\partial r_{i j}}{\partial r_{l \alpha}} r_{l \alpha} .
\end{aligned}
$$

From Eq. (A5) follows the relation

$$
\sum_{l=1}^{N} \frac{\partial r_{i j}}{\partial r_{l \alpha}} r_{l \alpha}=r_{i j}^{-1} r_{i j \alpha}^{2}
$$

Insertion of Eq. (C2) into Eq. (C1) yields

$$
W_{i \alpha}=\frac{1}{2} \sum_{j \neq i} \phi^{\prime}\left(r_{i j}\right) r_{i j \alpha}^{2} r_{i j}^{-1} .
$$

From Eq. (4.9) and Eq. (3.19), which gives $u_{e}$, we immediately have

$$
W_{e \alpha}=\frac{1}{2} \sum_{j \neq i} \phi^{\prime}\left(r_{i j}\right) r_{i j \alpha}^{2} r_{i j}^{-1},
$$

where $i$ stands for the centroid atom of element $e$.

From the expression for $f_{q}^{e}$ in Eq. (3.20), we obtain

$$
X_{e \gamma} \equiv \sum_{l=1}^{N} \frac{\partial f_{q}^{e}}{\partial r_{l \gamma}} r_{l \gamma}=\frac{k_{B} T}{2} \sum_{l=1}^{N} \frac{\partial \ln \left(\operatorname{det} \mathbf{D}_{e}\right)}{\partial r_{l \gamma}} r_{l \gamma},
$$

which we can write more explicitly as

$$
\begin{aligned}
X_{e \gamma}= & \frac{k_{B} T}{2 \operatorname{det}\left(\mathbf{D}_{e}\right)}\left[\left(D_{y y} D_{z z}-D_{y z}^{2}\right) S_{x x}^{(\gamma \gamma)}\right. \\
& +\left(D_{x x} D_{z z}-D_{x z}^{2}\right) S_{y y}^{(\gamma \gamma)}+\left(D_{x x} D_{y y}-D_{x y}^{2}\right) S_{z z}^{(\gamma \gamma)} \\
& +2\left(D_{x z} D_{y z}-D_{z z} D_{x y}\right) S_{x y}^{(\gamma \gamma)} \\
& +2\left(D_{x y} D_{y z}-D_{y y} D_{x z}\right) S_{x z}^{(\gamma \gamma)}+2\left(D_{x y} D_{x z}\right. \\
& \left.\left.-D_{x x} D_{y z}\right) S_{y z}^{(\gamma \gamma)}\right]_{e},
\end{aligned}
$$

where

$$
S_{\alpha \beta}^{(\gamma \gamma)} \equiv \sum_{l=1}^{N} \frac{\partial D_{\alpha \beta}}{\partial r_{l \gamma}} r_{l \gamma} .
$$

The subscript $e$ on $\mathbf{D}_{e}$ (which is dropped on matrix elements) and on the braces $[\cdots]_{e}$ in Eq. (C6) signifies that the associated dynamical quantity pertains to element $e$.

Substitution of Eq. (B4) for $D_{\alpha \beta}$ into Eq. (C7) gives

$$
\begin{aligned}
S_{\alpha \beta}^{(\gamma \gamma)}= & 24 \varepsilon \sigma^{-2} m^{-1} \sum_{j \neq i}\left\{\left[\sum_{l=1}^{N} \frac{\partial a_{i j}^{*}}{\partial r_{l \gamma}} r_{l \gamma}\right] r_{i j \alpha}^{*} r_{i j \beta}^{*}\right. \\
& +a_{i j}^{*}\left[\sum_{l=1}^{N} \frac{\partial r_{i j \alpha}^{*}}{\partial r_{l \gamma}} r_{l \gamma}\right] r_{i j \beta}^{*}+a_{i j}^{*} r_{i j \alpha}^{*}\left[\sum_{l=1}^{N} \frac{\partial r_{i j \beta}^{*}}{\partial r_{l \gamma}} r_{l \gamma}\right] \\
& \left.-\left[\sum_{l=1}^{N} \frac{\partial b_{i j}^{*}}{\partial r_{l \gamma}} r_{l \gamma}\right] \delta_{\alpha \beta}\right\} .
\end{aligned}
$$

From Eq. (B5) we have 


$$
\sum_{l=1}^{N} \frac{\partial a_{i j}^{*}}{\partial r_{l \gamma}} r_{l \gamma}=A_{i j}^{*} \sum_{l=1}^{N} \frac{\partial r_{i j}^{*}}{\partial r_{l \gamma}} r_{l \gamma}
$$

where

$$
A_{i j}^{*} \equiv-448 r_{i j}^{*-17}+80 r_{i j}^{*-11}
$$

and

$$
\sum_{l=1}^{N} \frac{\partial b_{i j}^{*}}{\partial r_{l \gamma}} r_{l \gamma}=B_{i j}^{*} \sum_{l=1}^{N} \frac{\partial r_{i j}^{*}}{\partial r_{l \gamma}} r_{l \gamma},
$$

where

$$
B_{i j}^{*} \equiv-28 r_{i j}^{*-15}+8 r_{i j}^{*-9} \text {. }
$$

From Eqs. (A4) and (A5) we deduce, respectively,

$$
\sum_{l=1}^{N} \frac{\partial r_{i j \alpha}^{*}}{\partial r_{l \gamma}} r_{l \gamma}=\sum_{l=1}^{N}\left(\delta_{i l}-\delta_{j l}\right) \delta_{\alpha \gamma} r_{l \gamma}^{*}=r_{i j \gamma}^{*} \delta_{\alpha \gamma}
$$

and

$$
\sum_{l=1}^{N} \frac{\partial r_{i j}^{*}}{\partial r_{l \gamma}} r_{l \gamma}=\sum_{l=1}^{N} r_{i j}^{*-1} r_{i j \gamma}^{*}\left(\delta_{i l}-\delta_{j l}\right) r_{l \gamma}^{*}=r_{i j}^{*-1} r_{i j \gamma}^{* 2} .
$$

Combining Eqs. (C8)-(C14), we arrive finally at

$$
\begin{aligned}
S_{\alpha \beta}^{(\gamma \gamma)}= & 24 \varepsilon \sigma^{-2} m^{-1} \sum_{j \neq i}\left[A_{i j}^{*} r_{i j \alpha}^{*} r_{i j \beta}^{*} r_{i j \gamma}^{* 2} r_{i j}^{*-1}+a_{i j}^{*} r_{i j \beta}^{*} r_{i j \gamma}^{*} \delta_{\alpha \gamma}\right. \\
& \left.+a_{i j}^{*} r_{i j \alpha}^{*} r_{i j \gamma}^{*} \delta_{\beta \gamma}-B_{i j}^{*} r_{i j \gamma}^{* 2} r_{i j}^{*-1} \delta_{\alpha \beta}\right] .
\end{aligned}
$$

${ }^{1}$ S. Kohlhoff, P. Gumbsch, and H. F. Fischmeister, Philos. Mag. A 64, 851 (1991)

${ }^{2}$ E. B. Tadmor, M. Ortiz, and R. Phillips, Philos. Mag. A 73, 1529 (1996).

${ }^{3}$ V. B. Shenoy, R. Miller, E. B. Tadmor, R. Phillips, and M. Ortiz, Phys. Rev. Lett. 80, 742 (1998).

${ }^{4}$ E. B. Tadmor, G. S. Smith, N. Bernstein, and E. Kaxiris, Phys. Rev. B 59, 235 (1999).

${ }^{5}$ E. B. Tadmor, R. Phillips, and M. Ortiz, Int. J. Solids Struct. 37, 379
(2000)

${ }^{6}$ F. F. Abraham, J. Q. Broughton, N. Bernstein, and E. Kaxiris, Comput. Phys. 12, 538 (1998).

${ }^{7}$ R. Rudd and J. Q. Broughton, Phys. Rev. B 58, R5893 (1998).

${ }^{8}$ J. Q. Broughton, F. F. Abraham, N. Bernstein, and E. Kaxiris, Phys. Rev. B 60, 2391 (1999)

${ }^{9}$ R. E. Miller and E. B. Tadmor, J. Comput.-Aided Mater. Des. 9, 203 (2002).

${ }^{10}$ W. A. Curtin and R. E. Miller, Modell. Simul. Mater. Sci. Eng. 11, R33 (2003).

${ }^{11}$ R. E. Miller, Int. J. Multiscale Comp. Eng. 1, 57 (2003).

${ }^{12}$ H. S. Park and W. K. Liu, Comput. Methods Appl. Mech. Eng. 193, 1733 (2004).

${ }^{13}$ H. Zhou, R. Feng, D. J. Diestler, and X. C. Zeng, J. Chem. Phys. 123, 164109 (2005).

${ }^{14}$ Z.-B. Wu, D. J. Diestler, R. Feng, and X. C. Zeng, J. Chem. Phys. 119, 8013 (2003).

${ }^{15}$ M. P. Allen and D. J. Tildesley, Computer Simulation of Liquids (Clarendon, Oxford, 1989), Chap. 4.

${ }^{16}$ D. J. Diestler, Z.-B. Wu, and X. C. Zeng, J. Chem. Phys. 121, 9279 (2004).

${ }^{17}$ Z.-B. Wu, D. J. Diestler, R. Feng, and X. C. Zeng, J. Chem. Phys. 120, 6744 (2004).

${ }^{18}$ Z.-B. Wu, D. J. Diestler, and X. C. Zeng, J. Chem. Phys. 121, 8029 (2004)

${ }^{19}$ Z.-B. Wu, D. J. Diestler, and X. C. Zeng, Mol. Simul. 31, 811 (2005).

${ }^{20}$ V. B. Shenoy, R. Miller, E. B. Tadmor, D. Rodney, R. Phillips, and M. Ortiz, J. Mech. Phys. Solids 47, 611 (1999).

${ }^{21}$ L. J. Segerlind, Applied Finite Element Analysis (Wiley, New York, 1976), Chap. 3.

${ }^{22}$ J. Rickman and R. LeSar, Annu. Rev. Mater. Res. 32, 195 (2002).

${ }^{23}$ D. A. McQuarrie, Statistical Mechanics (Harper \& Row, New York, 1976), Chap. 13.

${ }^{24}$ T. Egami and V. Vitek, J. Non-Cryst. Solids 61, 499 (1984).

${ }^{25}$ D. N. Theodorou and U. W. Suter, Macromolecules 19, 379 (1986).

${ }^{26}$ H. Zhou, Ph.D. thesis, University of Nebraska-Lincoln, 2006.

${ }^{27}$ A. Okabe, B. Boots, and K. Sugihara, Special Tessellations: Concepts and Applications of Voronoi Diagrams (Wiley, New York, 1992).

${ }^{28}$ M. Zhou, Proc. R. Soc. London, Ser. A 459, 2347 (2003).

${ }^{29}$ W. H. Press, S. A. Teukolsky, W. T. Vetterling, and B. P. Flannery, Numerical Recipes in FORTRAN 77: The Art of Scientific Computing, 2nd ed. (Cambridge University Press, Cambridge, 1996), Chap. 10.

${ }^{30} \mathrm{~W}$. Bollmann, Crystal Defects and Crystalline Interfaces (Springer, Berlin, 1970), Chap. 12. 